Kansas State University Libraries

New Prairie Press

\title{
USING RANDOM SAMPLING TO ESTIMATE INSECT COUNTS AS RESPONSE SURFACES INVOLVING SPACE AND TIME
}

Benjamin G. Mullinix

Glynn Tillman

Follow this and additional works at: https://newprairiepress.org/agstatconference

Part of the Agriculture Commons, and the Applied Statistics Commons

\section{c) (1) $\Theta$}

This work is licensed under a Creative Commons Attribution-Noncommercial-No Derivative Works 4.0 License.

\section{Recommended Citation}

Mullinix, Benjamin G. and Tillman, Glynn (2006). "USING RANDOM SAMPLING TO ESTIMATE INSECT COUNTS AS RESPONSE SURFACES INVOLVING SPACE AND TIME," Conference on Applied Statistics in Agriculture. https://doi.org/10.4148/2475-7772.1123

This is brought to you for free and open access by the Conferences at New Prairie Press. It has been accepted for inclusion in Conference on Applied Statistics in Agriculture by an authorized administrator of New Prairie Press. For more information, please contact cads@k-state.edu. 


\title{
Using Random Sampling to Estimate Insect Counts as Response Surfaces Involving Space and Time
}

\author{
Benjamin G. Mullinix, Research Statistician \\ and \\ Glynn Tillman, Research Entomologist \\ University of Georgia, Tifton Campus \\ Tifton, GA 31793-0748 \\ and
}

USDA-ARS Crop Protection \& Management Research Lab

Tifton, GA 31793-0748

Presented at the

$18^{\text {th }}$ Annual KSU Conference on

Applied Statistics in Agriculture

April 30 - May 2, 2006

Holiday Inn

Manhattan, Kansas 


\begin{abstract}
In fall 2000, an on-farm sustainable agricultural research project was established for cotton (Gossypium hirstum L.) in Tift County, Georgia. Twenty fields that were to be planted to cotton in 2001 were identified which were approximately 5 to 10 acres in size. Four randomly selected fields were assigned to each of five cover crops: 1) cereal rye (Secale cereale L.); 2) crimson clover (Trifolium incarnatum L.); 3) legume mixture of balansa clover (T. michelianum Savi), crimson clover, and hairy vetch (Vicia villosa Roth); 4) previous legume mixture plus cereal rye; and 5) no cover crop (fallow) in conventionally tilled fields. Cotton was planted in two rows (36 in apart) on six foot beds. A strip was burned out in each row in the four cover treatments using paraquat so cotton could be planted. In the spring, insect counts were determined using sweep nets in the covers and when the cotton was small. Cotton plants from emergence to four weeks old were not sampled since the sweep net could break the fragile cotton plants. Five more weeks of sweep net data were collected from cotton. Insect samples after this involved whole plants since the cotton was too big for the sweep net to be effective. Each field was divided into 24 x 24 foot sample areas beginning at the center of the field. Each week for 14 or 15 weeks, 21 samples were obtained from each field. Comprising the 21 random samples were one sample from the four center plots, one sample from each of the four sides, and four samples from each of the four quadrants. Thus throughout the season, a five acre field could have most of the plots sampled at least once. Larger fields saw a smaller percentage of all the plots sampled. No interior plot excluding the center and edges was sampled a second time until every plot had been sampled once. A response surface was fitted for the weekly data for each field. As would be expected, high densities of insects resulted in a significant fit.
\end{abstract}




\section{INTRODUCTION}

One of the most dynamic species to sample for is insects. They are ubiquitous in that they occur on every known plant species, whether grown for food or fiber, or classified as a garden flower, wildflower, or weed. Numerous species of insects are found on cotton (Gossypium hirsutum L.) and are classified either as a pest, parasitoid, or predator (Tillman et al., 2004). Pests include these Heliothines: The TBW (tobacco bud worm), Heliothis virescens (F.), and CEW (corn ear worm), Helicoverpa zea (Boddie). Other pests include the TPB (tarnished plant bug), Lygus lineolaris (Palisot de Beauvois), SGSB (southern green stink bug), Nezara viridula, BSB (brown stink bug), Euschistus servus (Say), and GSB (green stink bug), Acrosternum hilare (Say). Parasitoid insects parasitize pest insects, but do not bother the cotton otherwise. Predatory insects feed on other insects. Some feed primarily on insects. Others feed on the cotton plant as well. These include the big-eyed bug, Geocoris punctipes (Say); pirate bug, Orius insidiosus (Say); red imported fire ant, Solenopsis invicta Buren; and four lady bugs: convergent lady beetle, Hippodamia convergens Guerin-Meneville; sevenspotted lady beetle, Coccinella septempunctata L.; ladybird beetle, Coleomegilla maculata (DeGeer); and multicolored Asian lady beetle, Harmonia axyridis (Pallas).

Cotton is grown primarily for the cotton fiber that is produced by the seed. During the last half of the 20-th century, pressing cotton seed for cotton seed oil became an international business (all vegetable oils now rank among the ten most traded commodities on the world market) (Cherry et al., 1986). The pulp that is left after pressing is often fed to cattle as well as other livestock. However, whole cottonseed is also fed to cattle. Cattle will consume the cotton 
stalks left after machine picking and will even eat the cotton bolls that are lost during harvesting. Cotton is a multi-billion dollar industry in the United States. It is an even bigger industry outside the U.S.

Cotton is a tropical plant that dies when the air temperature drops below 32 degrees F ( 0 C) for any appreciable length of time (Mauney, 1986). Across the southern portion of the U.S., cotton is generally planted in May or early June and is prepared for harvest in September or October by spraying a herbicide-like chemical that causes the leaves to drop off the plant (Delouche, 1986). Cotton is harvested by spindle pickers in the southeast and in Arizona and California, while it is harvested by stripper pickers in Texas. Some stripper pickers are used in the Mississippi Delta region to recover the cotton left on the plant after the first pass with a spindle picker. Cotton which is stained by the green leaves is docked heavily at the cotton gin since it requires bleaching to restore its whiteness. Colored cotton is native to Peru, Bolivia, and northern Chile (Cherry et al., 1986). The high plains region of western Texas, the first killing frost of the season is generally used to kill the plant and this causes the leaves to drop off.

Over half of all the cotton grown in this country is irrigated (including that grown in Georgia, Texas, Arizona, and California). This makes the cotton plant more attractive to the insects that have learned to feed on the various parts of the cotton plant. Since the cotton boll is the most desired part of the plant from the standpoint of value to the grower, a lot of money is spent to keep insects off the boll. However, the current market price for cotton is around forty cents per pound of lint cotton (the fiber removed from the seed during ginning). This makes it increasingly unreasonable to apply expensive chemicals to the bolls to keep the insects from attacking the boll while it is developing. Research at Tifton has demonstrated that under typical 
conditions the boll requires approximately 49 days from bloom until it is ready for harvest during the hot summer (Bednarz, personal communication). Cooler conditions lengthen the time for development. In an effort to reduce chemical pesticide usage, various cover crops were proposed as a means of attracting predatory insects of cotton before the cotton is planted, hoping they would remain nearby so as to attack the insect pests of cotton when they arrive. A sustainable agriculture grant was proposed and was funded as part of a larger grant proposal to be administered on-farm in hopes of demonstrating an alternative to chemical pesticide use (these grants also support organic farming).

\section{METHODS AND MATERIALS}

A detailed description of the experiment can be found in Tillman et al. (2004). Each field was drawn to scale from the Tift County Soil Survey. All rectangular fields were divided into four quadrants. Distances were measured to establish the size of each field to determine how many 24x24 foot sampling areas there were. The paper map was then completed to reflect the total number of 24x24 foot sampling areas. In each quadrant the four center plots were marked off first and labeled (1-4). The four edges of each quadrant were then marked off and numbered beginning from right side for the bottom edge or upper right corner for the right edge, and from the left side for the top edge or lower left corner for the left edge. The remaining plots were numbered beginning at the corner opposite the center and proceeded row by row. Irregular fields were marked off in quadrants as best as could be done. Using a backpack GPS unit, coordinates were established for each quadrant in each field. An algorithm was developed to assign 
coordinates to each plot. A beeper on the GPS unit would signal when the correct plot was found. A sweep net was used to catch the insects present on the plants. The insects were killed with $\mathrm{CO}_{2}$ gas and placed in a sampling container for later identification in the laboratory. This procedure was repeated for every plot that had been randomly selected to be sampled each week.

A SAS (SAS, 2002) program using Proc PLAN was constructed to provide the randomly selected plots that were to be sampled each week in each field (Cochran and Cox, 1957). One of the four center plots were selected each week for each field to be sampled. One plot from each of the four edges was randomly selected each week for each field to be sampled. Four to 14 plots were randomly selected from each quadrant each week for each field to be sampled. The number of plots sampled for each field varied from 21 to 61 depending on the number of student workers available and on time constraints. All twenty fields' randomly chosen numbers were entered in to a special file format to operate in backpack GPS unit that was plugged into a laptop PC. Out in the field, the two people assigned would follow a map showing where the plots to be sampled this week were located. The GPS unit would beep when the 24 x 24 foot plot's center was found. This was possible since the GPS coordinates for a corner of the field was loaded into the PC and each plot's center was automatically determined. The data were entered into a PC in an Excel worksheet. The worksheet's contents were read directly into the SAS analysis program using the dde (dynamic data exchange) feature in the data step of SAS. Once the data was brought into SAS, the data were sorted and merged with a master file that contained every plot that could have been sampled for every field for every week. After merging and then by sorting by cover crop and sampling week, Proc MIXED (SAS,2002) was used to analyze the data to fit a response surface model that described the mean of the four fields with the same cover crop treatment. The 
model used the following fixed effects: tLRdistance (distance left to right in units of 24 feet where the first plot either left or right of the center had a value twelve, the values were divided by 10, values to the left were negative); tUDdistance (distance up [north] to down in units of 24 feet where the first plot either above or below of the center had a value twelve, the values were divided by 10, values to the below were negative); tLRdistance*tLRdistance;

tUDdistance*tUDdistance; and tLRdistance*tUDdistance. The model statement option 'OUTP=data-set-name' was used to create an output file with the predicted values for every plot in every field. This new output file was divided into the five cover crop treatments and two sampling week groups: 1) when the cover crops were present (weeks 1-10), and 2) when the cotton was present (weeks 14-18). A second Proc MIXED analysis was used to analyze the data. The model used the following effects: mSampleWeek (either weeks 1-10 with 5.5 subtracted from each week value [suggested by Draper \& Smith, 1981] for the cover weeks; or weeks 14-18 with 16 subtracted from each week value for the cotton weeks); mSampleWeek*mSampleWeek; tLRdistance (as described previously); tLRdistance*tLRdistance; tUDdistance (as described previously); tUDdistance*tUDdistance; mSampleWeek*tLRdistance; SampleWeek*tUDdistance; and tLRdistance*tUDdistance. The solution betas were used in a third SAS program to construct the three dimensional set of data comprised of sample week, leftright distance, and up-down distance. Proc G3D was used to construct response surfaces depicting the values associated with these three analysis variables (Total Insect Pests [destroy cotton plant], Total Insect Predators [attacks insect pests], and Total Insect Parasitoids [attack the Heliothines $\{$ worms $\}]$ ) on a weekly basis (195 of these graphs were produced). Two graphs were placed on a landscaped page which resulted in 105 landscaped pages. Results for Crimson 
Clover cover crop will be presented.

\section{RESULTS AND DISCUSSION}

Two examples will be presented. Figure 1 shows the shape of Branch's field where the cover crop Blend+Rye was planted. The four center plots were labeled A-D. The edges were

numbered as well. Notice how the interior plots were numbered beginning with the four corners and proceeding towards the center. Table 1 contains the SAS statements used produce these random plot numbers for Branch's Blend+Rye. The first factors request is to get the center plot to sample each week. The next four factors requests are to get one plot from the edge plots for each week. The remaining eight factors requests are to get 13 plots each week from two of the larger quadrants, while 11 plots each week from the two smaller quadrants. It takes about 12 weeks to sample every plot once from the larger quadrants, while it takes about 13 weeks to sample every plot from the smaller quadrants.

Table 3 shows the results of the analysis of the data collected that was classified as Total Insect Pests (data are the predicted values computed for each 24 x 24 foot plot area in every field) before cotton is planted. The analysis performed by Proc MIXED (SAS, 2002) is divided into two parts, random and fixed effects designated as either R or F, respectively. Variances for random effects are components of variance and are present if the contribution to variance exceeds the total variance associated with all random effects below it (Residual is an exception). A multiplier is associated with each variance component, except for Residual Error which has a multiplier of one. Although the five cover crop treatments had a non-zero and non-negative 
value, its contribution is not as important. Variance components for DLR(CC) [read DLR within Cover crop] and DUD(CC) are for the unused SS and DF left after fitting the fixed effects for DLR linear and quadratic and for DUD linear and quadratic which are significant contributions to variance. Sample Week had a variance component of zero which means that the portion left over after fitting the fixed effects of linear and quadratic was not large enough. The interaction of CC and Sample Week as a variance component had a significant contribution to variance. It was not expected that there would be a linear effect due to Sample Week since insects in general are known for increasing population numbers when the crop is attractive and then decreasing when the attractiveness subsides. The quadratic effect due to Sample Week is extremely significant (F ge 126.62). Linear and quadratic effects of DLR was significant, but only the linear effect of DUD was significant. Only the interaction of linear effect of Sample Week with the linear effect of DUD was highly significant (F ge 133.76). The interaction of linear effect of DLR with the linear effect of DUD was highly significant (F ge 193.40). The three way interaction of the linear effects of DLR, DUD, and Sample Week was significant. Table 4 shows the results of the Proc MIXED analysis of the predicted data from the twenty fields after cotton had been planted. The big surprise was the pronounced linear trend associated with Sample Week. However, there was no three way interaction. Table 5 shows the results of the Proc MIXED analysis of the predicted data from the twenty fields before cotton is planted for Total Insect Predators. The analysis is similar to Total Insect Pests which is the reason for the cover crops originally. This was certainly gratifying to see that the predators showed up because the insect pests were there. It is suspected that the attractiveness of the cover crops is what brought both pests and predators since there is a substantial amount of food present. Table 6 shows the 
results of the Proc MIXED analysis of the predicted data from the twenty fields after cotton had been planted. The biggest surprises were the presence of a linear trend associated with Sample Week and the amount of interaction of linear Sample Week with both DLR and DUD. However, there was no significant three way interaction. Table 7 shows the results of the Proc MIXED analysis of the predicted data from the twenty fields before cotton is planted for Total Insect Parasitoids. The analysis is similar to Total Insect Pests which is the reason for the cover crops originally. This was certainly gratifying to see that the parasitoids showed up because the insect pests were there, especially TBW and CEW. Table 8 shows the results of the Proc MIXED analysis of the predicted data from the twenty fields after cotton had been planted. The big surprise was the amount of interaction of linear Sample Week with both DLR and DUD. There was a significant three way interaction.

Figure 2a-e show the results of the weekly sampling transformed using a response surface methodology as described in Cochran and Cox (1957). Crimson clover produces a head of crimson colored tiny blooms arrayed in a bell shape. Insects love the plentiful supply of nectar and pollen available. There are tens of thousands of these plants in an acre. Cotton insect pest counts were near zero at week 1, but began to climb steadily towards week 5 where surface lies near a value of 14 . This level is maintained through week 7. The level begins to decline towards zero by week 10. Cotton is planted at about this time. Weekly sampling resumes at week 14 when the cotton is sturdy enough (about 21 after germination) to withstand the sweep net used to collect the insects. Insect pests appear to be the most numerous during week 14 (Figure 3a-c) when the plant is rapidly growing and plenty of food is traveling back and forth between the leaves and stems and the roots. 
The purpose of the research is to determine if the insect predators are following the cotton insect pests into the cotton fields. Figure 4a-e shows the level of insect predators from week 1 through week 10 . The greatest numbers occur during weeks 4 through 8 , at count of approximately six per plot. This demonstrates that the peak count occurred at the same time as the peak count of the insect pests. This was the hypothesized value of the various cover crops. After the cotton was up and growing, the number of insect predators was above three per plot for all five weeks of sweep net sampling (Figure 5a-c). Two of the insect pests are members of the Heliothine family: TBW (tobacco bud worn) and CEW (corn ear worm).

A parasitoid is small wasp that punctures their host and deposits an egg inside of it. Parasitoids attack Heliothine worms. Figure 6a-e depict the levels of parasitized worms found in the sweep net samples. Their numbers peaked during weeks 4 through 6 which is the same time that these two insect pests were found. They were present as well during the sampling of the young, growing cotton plants during weeks 14 to 18, but not in as great of numbers.

\section{CONCLUSIONS}

The data originally analyzed for presentation at a meeting of various groups with SARE grants seemed to indicate that the insect predators seemed to lag at least a week behind the insect pests (Tillman, personal communication). However, after obtaining the predicted values from the Proc MIXED analysis of each field's data and putting these predicted values together for a large analysis, graphical representations of the insect pest and predator data began to show that they arrived at nearly the same time as the cover crops. This was justification enough for the amount of work put into sampling so many fields for all those weeks. The graphs did show that there 
seemed to be more activity along the edges of the fields than previously believed. Hence, a new grant proposal has been submitted for sampling fewer fields but still studying cover crops. Other field work since has shown that the choice of crop planted adjacent or close to cotton has a huge impact on the numbers of insects found in cotton. 


\section{REFERENCES}

Cherry, J. P., R. J. Kohel, L. A. Jones, and W. H. Powell. 1986. Chapter 37. Food and feeding quality of cottonseed. In J. R. Mauney \& J. M. Stewart (Eds.) Cotton Physiology. Memphis, TN: The Cotton Foundation.

Cochran, W.G. and Cox, G.M. 1957. Experimental designs. John Wiley \& Sons, New York.

Draper, N.R. and H. Smith. 1981. Applied regression analysis ( $2^{\text {nd }}$ ed.). John Wiley \& Sons, New York.

Delouche, J. C. 1986. Chapter 32. Post-harvest factors affecting seed quality. In J. R. Mauney \& J. M. Stewart (Eds.) Cotton Physiology. Memphis, TN: The Cotton Foundation.

Mauney, J. R. 1986. Chapter 2. Vegetative growth and development of fruiting sites. In J. R. Mauney \& J. M. Stewart (Eds.) Cotton Physiology. Memphis, TN: The Cotton Foundation.

SAS Institute, Inc. 2002. SAS/C OnlineDoc ${ }^{\mathrm{TM}}$, Release 9.1. Cary, NC: SAS Institute, Inc.

Tillman G., H. Schomberg, S. Phatak, B. Mullinix, S. Lachnight, P. Timper and D. Olson. (2004). Influence of cover crops on insect pests and predators in conservation tillage cotton. J. Econ. Entomol. 97(4):1217-1232. 


\section{Acknowledgments}

Thanks is given to Ryan Branch, Brian Ponder, and Grady Thompson for providing the fields. Thanks is also expressed to professors Steve Brown, Glen Harris, Phillip Roberts, and Glen Rains for technical help provided for managing the cotton. Thanks is given to Penny Tapp, Kristie Graham, and Ron Dozier for helping in collecting the insect samples and work study students from Abraham Baldwin Agricultural College. 
Figure 1. A drawing showing the shape of Branch’s field where the cover crop, Blend+Rye, was planted with the 24 x $24 \mathrm{ft}$ plots numbered.

\section{Branch Blend+Rye}

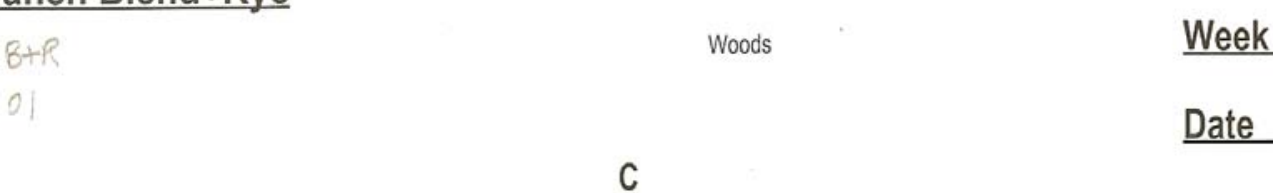

Quad 2

Quad 4

\begin{tabular}{|c|c|c|c|c|c|c|c|c|c|c|c|c|c|c|c|c|c|c|c|c|c|c|c|c|c|c|c|}
\hline 25 & 1 & 2 & 3 & 4 & 5 & 6 & 7 & 8 & 9 & 10 & 11 & 12 & 13 & 14 & 15 & 16 & 17 & 18 & 19 & 20 & 21 & $22 \quad 2$ & 23 & & 25 & & \\
\hline 24 & 1 & 2 & 3 & 4 & 5 & 6 & 7 & 8 & 9 & 10 & 11 & 12 & 13 & 13 & 12 & 11 & 10 & 9 & 8 & 7 & & 5 & 4 & 3 & 2 & 1 & \\
\hline 23 & 14 & 15 & 16 & 17 & 18 & 19 & 20 & 21 & 22 & 23 & 24 & 25 & 26 & 26 & 25 & 24 & 23 & 22 & 21 & 20 & 19 & 18 & 17 & 16 & 15 & 14 & \\
\hline 22 & 27 & 28 & 29 & 30 & 31 & 32 & 33 & 34 & 35 & 36 & 37 & 38 & 39 & 39 & 38 & 37 & 36 & 35 & 34 & 33 & 32 & 313 & 30 & 29 & 28 & 27 & \\
\hline 21 & 40 & 41 & 42 & 43 & 44 & 45 & 46 & 47 & 48 & 49 & 50 & 51 & 52 & 52 & 51 & 50 & 49 & 48 & 47 & 46 & 45 & \begin{tabular}{l|l}
44 & 4 \\
\end{tabular} & 43 & 42 & 41 & 40 & \\
\hline 20 & 53 & 54 & 55 & 56 & 57 & 58 & 59 & 60 & 61 & 62 & 63 & 64 & 65 & 65 & 64 & 63 & 62 & 61 & 60 & 59 & 58 & \begin{tabular}{l|l}
57 & 5
\end{tabular} & 56 & 55 & 54 & 53 & \\
\hline 19 & 66 & 67 & 68 & 69 & 70 & 71 & 72 & 73 & 74 & 75 & 76 & 77 & 78 & 78 & 77 & 76 & 75 & 74 & 73 & 72 & 71 & 706 & 69 & 68 & 67 & 66 & \\
\hline 18 & 79 & 80 & 81 & 82 & 83 & 84 & 85 & 86 & 87 & 88 & 89 & 90 & 91 & 91 & 90 & 89 & 88 & 87 & 86 & 85 & 84 & \begin{tabular}{l|l}
83 & 8
\end{tabular} & 82 & 81 & 80 & 79 & \\
\hline 17 & 92 & 93 & 94 & 95 & 96 & 97 & 98 & 99 & 100 & 101 & 102 & 103 & 104 & 104 & 103 & 102 & 101 & 100 & 99 & 98 & 97 & 969 & 95 & 94 & 93 & 92 & \\
\hline 16 & 105 & 106 & 107 & 108 & 109 & 110 & 111 & 112 & 113 & 114 & 115 & 116 & 117 & 117 & 116 & 115 & 114 & 113 & 112 & 111 & 1101 & 1091 & 108 & 107 & 1061 & 105 & 10 \\
\hline 15 & 118 & 119 & 120 & 121 & 122 & 123 & 124 & 125 & 126 & 127 & 128 & 129 & 130 & 130 & 129 & 128 & 127 & 126 & 125 & 124 & 1231 & $\begin{array}{ll}122 & 1 \\
\end{array}$ & 121 & 120 & 119 & 118 & 11 \\
\hline 14 & 131 & 132 & 133 & 134 & 135 & 136 & 137 & 138 & 139 & 140 & 141 & 142 & 143 & 143 & 142 & 141 & 140 & 139 & 138 & 137 & 136 & 1351 & 134 & 1331 & 1321 & 131 & 12 \\
\hline 13 & 144 & 145 & 146 & 147 & 148 & 149 & 150 & 151 & 152 & 153 & 154 & 155 & 2 & 4 & 155 & 154 & 153 & 152 & 151 & 150 & 149 & \begin{tabular}{l|l}
148 & 1
\end{tabular} & 147 & 146 & 1451 & 144 & 13 \\
\hline 12 & 131 & 132 & 133 & 134 & 135 & 136 & 137 & 138 & 139 & 140 & 141 & 144 & 1 & 3 & 142 & 741 & & 10 & 138 & 13 & & & 121 & 132 & & 131 & 17 \\
\hline 11 & 118 & 119 & 120 & 121 & 122 & 123 & 124 & 125 & 126 & 127 & 128 & 129 & 130 & 130 & 129 & & 121 & 126 & 125 & & & & 121 & 120 & & & 15 \\
\hline 10 & 105 & 106 & 107 & 108 & 109 & 110 & 111 & 112 & 113 & 114 & 115 & 116 & 117 & 117 & 116 & & & & 112 & & & 109 & 108 & 107 & 106 & 105 & 16 \\
\hline 9 & 92 & 93 & 94 & 95 & 96 & 97 & 98 & 99 & 100 & 101 & 102 & & 104 & 104 & 103 & & 101 & 100 & 99 & 98 & 97 & 96 & 95 & 94 & 93 & 92 & 17 \\
\hline 8 & 79 & 80 & 81 & 82 & 83 & 84 & 85 & 86 & 87 & 88 & 89 & 90 & 91 & 91 & 90 & 89 & 88 & 87 & 86 & 85 & 84 & 83 & 82 & 81 & 80 & 79 & 18 \\
\hline 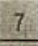 & 66 & 67 & 68 & 69 & 70 & 71 & 12 & 73 & 74 & 75 & 76 & 77 & 78 & 78 & 77 & 76 & 75 & 74 & 73 & 72 & 71 & 70 & 69 & 68 & 67 & 66 & 19 \\
\hline 0 & 53 & 54 & 55 & 56 & 57 & 58 & 59 & 60 & 61 & 62 & 63 & 64 & 65 & 65 & 64 & 63 & 62 & 61 & 60 & 59 & 58 & 57 & 56 & 55 & 54 & 53 & 20 \\
\hline 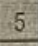 & 40 & 41 & 42 & 43 & 44 & 45 & 46 & 47 & 48 & 49 & 50 & 51 & 52 & 52 & 51 & 50 & 49 & 48 & 47 & 46 & 45 & 44 & 43 & 42 & 41 & 40 & 21 \\
\hline 4 & 27 & 28 & 29 & 30 & 31 & 32 & 33 & 34 & 35 & 36 & 37 & 38 & 39 & 39 & 38 & 37 & 36 & 35 & 34 & 33 & 32 & 31 & 30 & 29 & 28 & 27 & 22 \\
\hline 3 & 14 & 15 & 16 & 17 & 18 & 19 & 20 & 21 & 22 & 23 & 24 & 25 & 26 & 26 & 25 & 24 & 23 & 22 & 21 & 20 & 19 & 18 & 17 & 16 & 15 & 14 & 23 \\
\hline 2 & 1 & \begin{tabular}{|l|}
2 \\
\end{tabular} & 3 & 4 & \begin{tabular}{|l|}
5 \\
\end{tabular} & \begin{tabular}{l|l|}
6 \\
\end{tabular} & 7 & 8 & 9 & 10 & 11 & 12 & 13 & 13 & 12 & \begin{tabular}{|l|}
11 \\
\end{tabular} & 10 & \begin{tabular}{|l|}
9 \\
\end{tabular} & \begin{tabular}{l|l|}
8 \\
\end{tabular} & & 6 & \begin{tabular}{l|l|}
5 & \\
\end{tabular} & 4 & 3 & 2 & 1 & 2 \\
\hline & 26 & 25 & 24 & 23 & 22 & 21 & 20 & \begin{tabular}{l|l|}
19 \\
\end{tabular} & 18 & 17 & 16 & 15 & 14 & 13 & \begin{tabular}{l|l|}
12 \\
\end{tabular} & 11 & 10 & 9 & 8 & 7 & 6 & 5 & 4 & 3 & 2 & 1 & \\
\hline
\end{tabular}


Table 1. SAS program statements used to produced the needed random numbers to do weekly sampling at the Branch’s Blend+Rye Field.

title 'G.Tillman 2002: Sampling Plan';

title 'For 20 Cotton Fields with 16 Weeks of Plant Samples';

title 'File: CoverCrop2001';

proc plan; title2 'Field: Branchs Blend+Rye (B+R 01)';

title3 'Use 1 Number/Week';

factors Week=16 ordered PlotC=1 of 4; * Center;

factors Week=16 ordered PlotS=1 of 26; * S edge;

factors Week=16 ordered PlotW=1 of 25; * W edge;

factors Week=16 ordered PlotN=1 of 26; * N edge;

factors Week=16 ordered PlotE=1 of 25; * E edge;

proc plan; title3 'Use 13 Numbers/Week';

factors Plot=130 of 155; * NW quadrant;

factors Plot=78 of 155; * NW quadrant;

factors Plot=130 of 155; * NE quadrant;

factors Plot=78 of 155; * NE quadrant;

proc plan; title3 'Use 11 Numbers/Week';

factors Plot=110 of 142; * SW quadrant;

factors Plot=66 of 142; * SW quadrant;

factors Plot=110 of 142; * SE quadrant;

factors Plot=66 of 142; * SE quadrant;

run; 
Table 2. Output from the SAS program statements in Table 1 showing the random numbers to do weekly sampling at the Branch’s Blend+Rye Field.

File: CoverCrop2001

Field: Branchs Blend+Rye (B+R 01)

Use 1 Number/Week

The PLAN Procedure

Factor Levels Order Levels Order Levels Order Levels Order Levels Order

$\begin{array}{llllll}\text { Week } & 14 \mathrm{O} & 15 \mathrm{O} & 16 \mathrm{O} & 17 \mathrm{O} & 18 \mathrm{O}\end{array}$

$\begin{array}{llllll}\text { PlotC } & 4 \mathrm{R} & 26 \mathrm{R} & 26 \mathrm{R} & 26 \mathrm{R} & 26 \mathrm{R}\end{array}$

Week -PlotC- -PlotS- -PlotW- -PlotN- $\quad$-PlotE-

\begin{tabular}{|c|c|c|c|c|c|}
\hline \multicolumn{6}{|c|}{$\begin{array}{l}\text { Table 2. Output from the SAS program statements in } \\
\text { weekly sampling at the Branch's Blend+Rye Field. }\end{array}$} \\
\hline \multicolumn{6}{|c|}{ Field: Branchs Blend+Rye (B+R 01) } \\
\hline \multicolumn{6}{|c|}{ Use 1 Number/Week } \\
\hline \multicolumn{6}{|c|}{ The PLAN Procedure } \\
\hline Factor & Levels Or & r Levels & rder Leve & Order Le & els Order Levels Order \\
\hline Week & $14 \mathrm{O}$ & $15 \mathrm{O}$ & 160 & $17 \mathrm{O}$ & $18 \mathrm{O}$ \\
\hline PlotC & $4 \mathrm{R}$ & $26 \mathrm{R}$ & $26 \mathrm{R}$ & $26 \mathrm{R}$ & $26 \mathrm{R}$ \\
\hline Week & -PlotC- & -PlotS- & -PlotW- & -PlotN- & -PlotE- \\
\hline 1 & 3 & 10 & 21 & 2 & 1 \\
\hline 2 & 1 & 14 & 8 & 1 & 10 \\
\hline 3 & 4 & 15 & 16 & 13 & 9 \\
\hline 4 & 1 & 1 & 12 & 4 & 14 \\
\hline 5 & 3 & 4 & 3 & 5 & 23 \\
\hline 6 & 3 & 7 & 11 & 10 & 11 \\
\hline 7 & 1 & 26 & 13 & 22 & 13 \\
\hline 8 & 3 & 6 & 16 & 16 & 21 \\
\hline 9 & 1 & 2 & 11 & 13 & 14 \\
\hline
\end{tabular}

\begin{tabular}{|c|c|c|c|c|c|}
\hline \multicolumn{6}{|c|}{$\begin{array}{l}\text { Table 2. Output from the SAS program statements in } \\
\text { weekly sampling at the Branch's Blend+Rye Field. }\end{array}$} \\
\hline \multicolumn{6}{|c|}{ Field: Branchs Blend+Rye (B+R 01) } \\
\hline \multicolumn{6}{|c|}{ Use 1 Number/Week } \\
\hline \multicolumn{6}{|c|}{ The PLAN Procedure } \\
\hline Factor & Levels Or & r Levels & rder Leve & Order Le & els Order Levels Order \\
\hline Week & $14 \mathrm{O}$ & $15 \mathrm{O}$ & 160 & $17 \mathrm{O}$ & $18 \mathrm{O}$ \\
\hline PlotC & $4 \mathrm{R}$ & $26 \mathrm{R}$ & $26 \mathrm{R}$ & $26 \mathrm{R}$ & $26 \mathrm{R}$ \\
\hline Week & -PlotC- & -PlotS- & -PlotW- & -PlotN- & -PlotE- \\
\hline 1 & 3 & 10 & 21 & 2 & 1 \\
\hline 2 & 1 & 14 & 8 & 1 & 10 \\
\hline 3 & 4 & 15 & 16 & 13 & 9 \\
\hline 4 & 1 & 1 & 12 & 4 & 14 \\
\hline 5 & 3 & 4 & 3 & 5 & 23 \\
\hline 6 & 3 & 7 & 11 & 10 & 11 \\
\hline 7 & 1 & 26 & 13 & 22 & 13 \\
\hline 8 & 3 & 6 & 16 & 16 & 21 \\
\hline 9 & 1 & 2 & 11 & 13 & 14 \\
\hline
\end{tabular}

4$$
5
$$

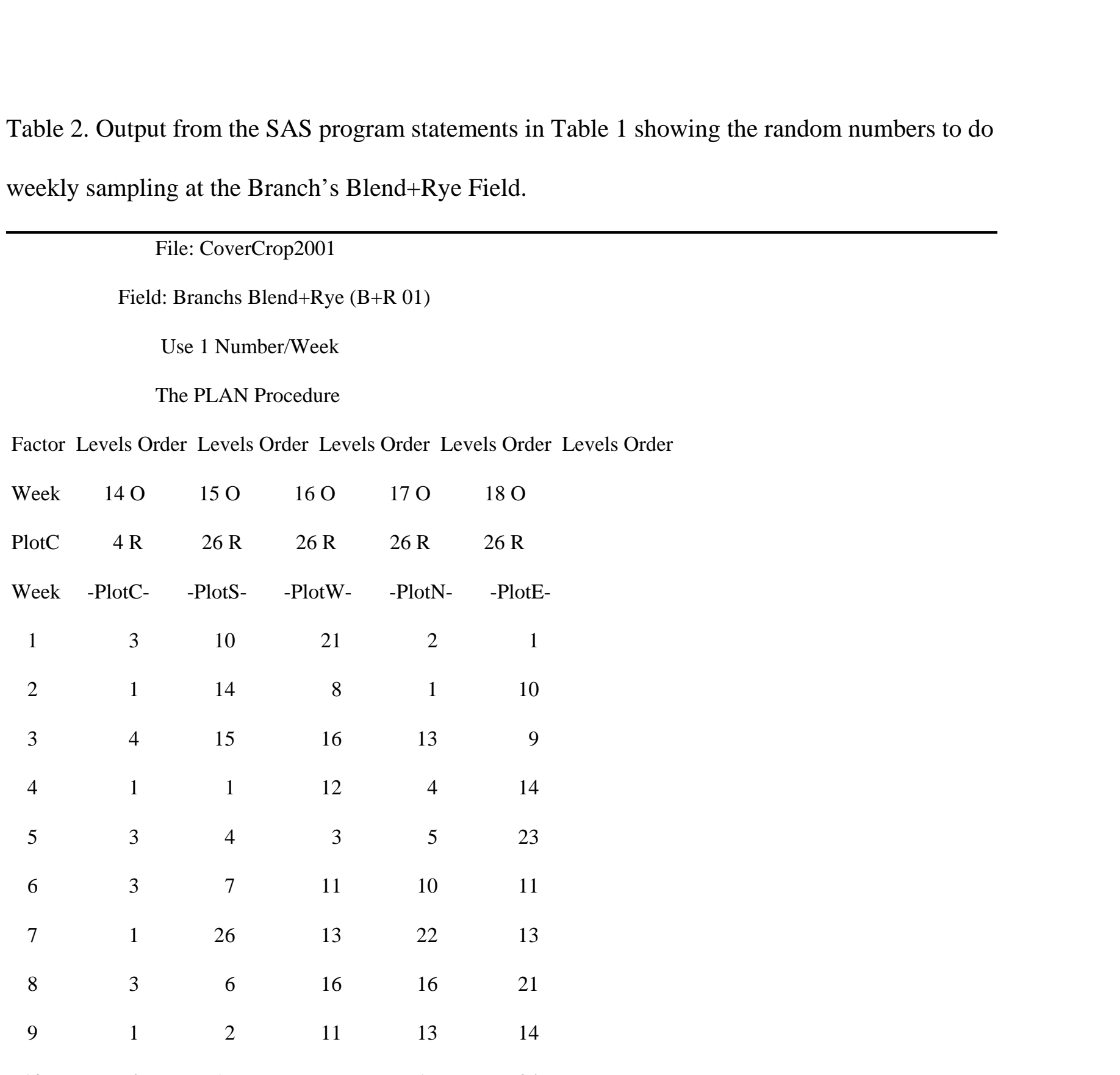

\begin{tabular}{|c|c|c|c|c|c|}
\hline \multicolumn{6}{|c|}{$\begin{array}{l}\text { Table 2. Output from the SAS program statements in } \\
\text { weekly sampling at the Branch's Blend+Rye Field. }\end{array}$} \\
\hline \multicolumn{6}{|c|}{ Field: Branchs Blend+Rye (B+R 01) } \\
\hline \multicolumn{6}{|c|}{ Use 1 Number/Week } \\
\hline \multicolumn{6}{|c|}{ The PLAN Procedure } \\
\hline Factor & Levels Or & r Levels & rder Leve & Order Le & els Order Levels Order \\
\hline Week & $14 \mathrm{O}$ & $15 \mathrm{O}$ & 160 & $17 \mathrm{O}$ & $18 \mathrm{O}$ \\
\hline PlotC & $4 \mathrm{R}$ & $26 \mathrm{R}$ & $26 \mathrm{R}$ & $26 \mathrm{R}$ & $26 \mathrm{R}$ \\
\hline Week & -PlotC- & -PlotS- & -PlotW- & -PlotN- & -PlotE- \\
\hline 1 & 3 & 10 & 21 & 2 & 1 \\
\hline 2 & 1 & 14 & 8 & 1 & 10 \\
\hline 3 & 4 & 15 & 16 & 13 & 9 \\
\hline 4 & 1 & 1 & 12 & 4 & 14 \\
\hline 5 & 3 & 4 & 3 & 5 & 23 \\
\hline 6 & 3 & 7 & 11 & 10 & 11 \\
\hline 7 & 1 & 26 & 13 & 22 & 13 \\
\hline 8 & 3 & 6 & 16 & 16 & 21 \\
\hline 9 & 1 & 2 & 11 & 13 & 14 \\
\hline
\end{tabular}

$\begin{array}{rrrrrr}10 & 4 & 17 & 7 & 16 & 24 \\ 11 & 2 & 25 & 16 & 1 & 7 \\ 12 & 4 & 6 & 20 & 15 & 2 \\ 13 & 3 & 20 & 25 & 2 & 6 \\ 14 & 3 & 20 & 22 & 5 & 13 \\ 15 & 2 & 8 & 21 & 12 & 14 \\ 16 & 1 & 16 & 2 & 5 & 11\end{array}$


Table 2 (continued)

\author{
The PLAN Procedure \\ Factor Select Levels Order \\ Plot $\quad 130 \quad 155$ Random \\ 4984513713412229641491431531191441021361173369104 \\ $35 \quad 18914780113493701511051391136612713286101146$ \\ $858111022124145 \quad 782144125 \quad 67 \quad 2 \quad 42 \quad 88 \quad 91546312983$ \\ 1031111169715141751071331009146407699241149668

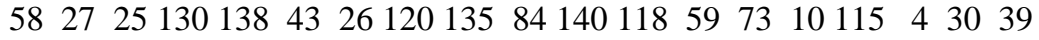

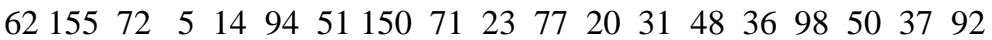 \\ $12109142196517 \quad 7987112126 \quad 3128 \quad 82148 \quad 16 \quad 13$
}

The PLAN Procedure

Factor Select Levels Order

Plot $\quad 78 \quad 155$ Random

$9138 \quad 887211474591413215 \quad 4 \quad 49661202157 \quad 64 \quad 42 \quad 12$

$69100 \quad 7106 \quad 38131355815039 \quad 2 \quad 6196101542614114583$

147122841181341375311982140451531271114950125142129

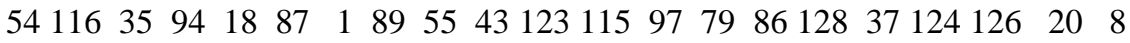

The PLAN Procedure

$\begin{array}{lccc}\text { Factor } & \text { Select } & \text { Levels } & \text { Order } \\ \text { Plot } & 130 & 155 \quad \text { Random }\end{array}$

116871149086148811077270149109137130209714715522

$521501137945129646748 \quad 841531104314312371 \quad 211518$

1031401171122154408814578126251466310638342628 
Table 2 (continued)

$6810274131111918959139531411995104 \quad 4 \quad 4914105122$

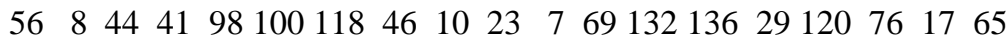

$9655119447119151153085154364214412513 \quad 37121124$

$135313373 \quad 62 \quad 8035128 \quad 9134 \quad 24 \quad 12 \quad 82 \quad 32142 \quad 66$

The PLAN Procedure

Factor Select Levels Order

Plot $\quad 78 \quad 155$ Random

1409473543628431423411774651112711013131101152

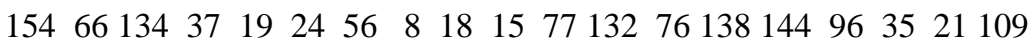

$10422618053145911315520278611648136124128 \quad 4146$

$106148 \quad 881471537950 \quad 6 \quad 1 \quad 5 \quad 83871351038 \quad 2657107133 \quad 108 \quad 23$

The PLAN Procedure

Factor Select Levels Order

Plot $\quad 110 \quad 142$ Random

23113119367857751188573124107315891109274160

$118744 \quad 4107710311510014213397213121647012761$

$1114913316 \quad 6212313225125138519411050 \quad 8129 \quad 1 \quad 45 \quad 76$

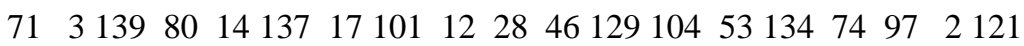

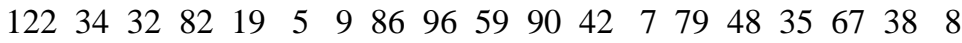

$66 \quad 6 \quad 89126181124083993712030130136102$

The PLAN Procedure

Factor Select Levels Order 
Table 2 (continued)

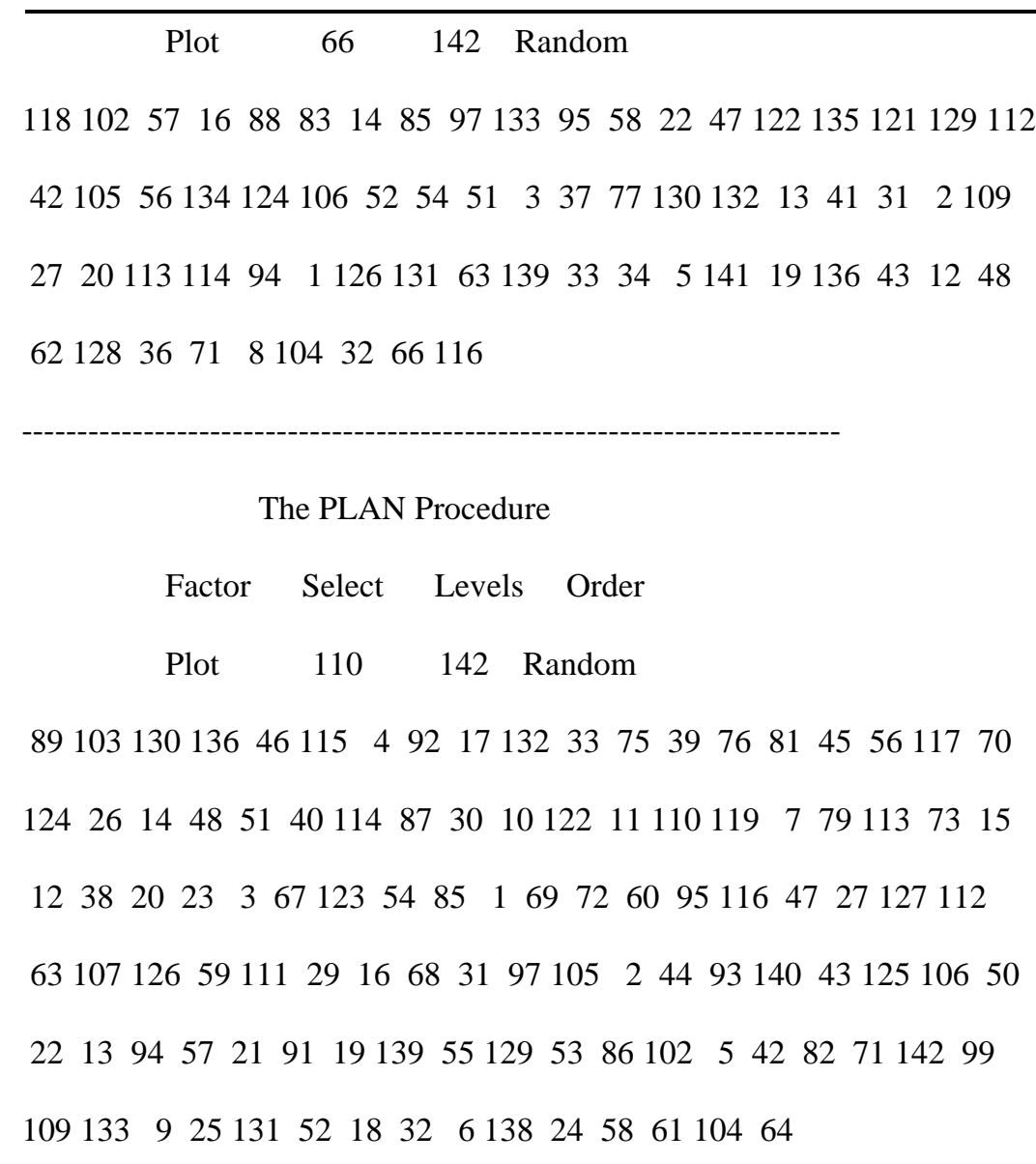

The PLAN Procedure

Factor Select Levels Order

Plot $\quad 66 \quad 142$ Random

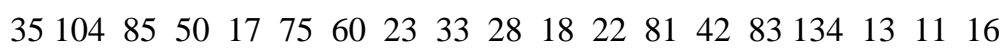

$11711112212166 \quad 53261261025911262 \quad 73127 \quad 8 \quad 6437118131$

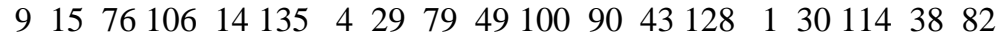

1191293105125931214044 


\section{Cover Crop Effect on Numbers of Insects on Cotton \\ Sompletime=Cover Crop, Cover Crop=Crimson Clover \\ PTPe = Predicted Totol Pests \\ Sompletherk =1}

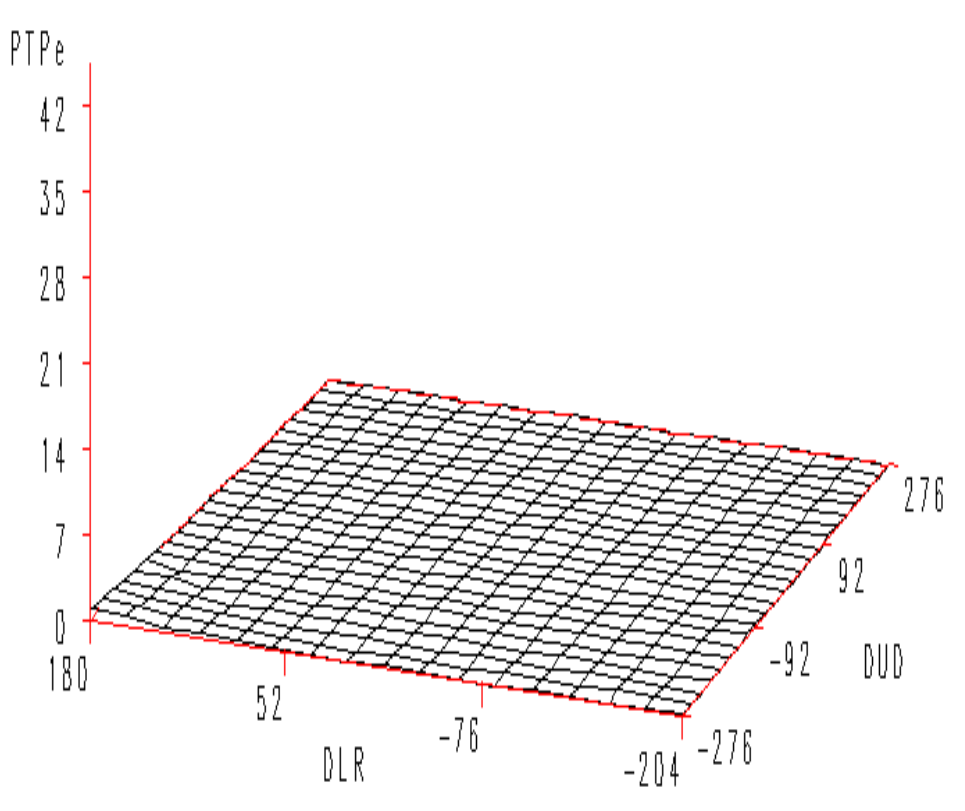

$D L R=D$ istance $(\mathrm{ft})$ Left to Right, DUD $=$ Distance $(\mathrm{ft})$ Up to Down, Up $=$ North

\section{Cover Crop Effect on Numbers of Insects on Cotton \\ Sompletime=Cover Crop, Cover Crop=Crimson Clover PTPe = Predicted Totol Pests Sompletheek $=2$}

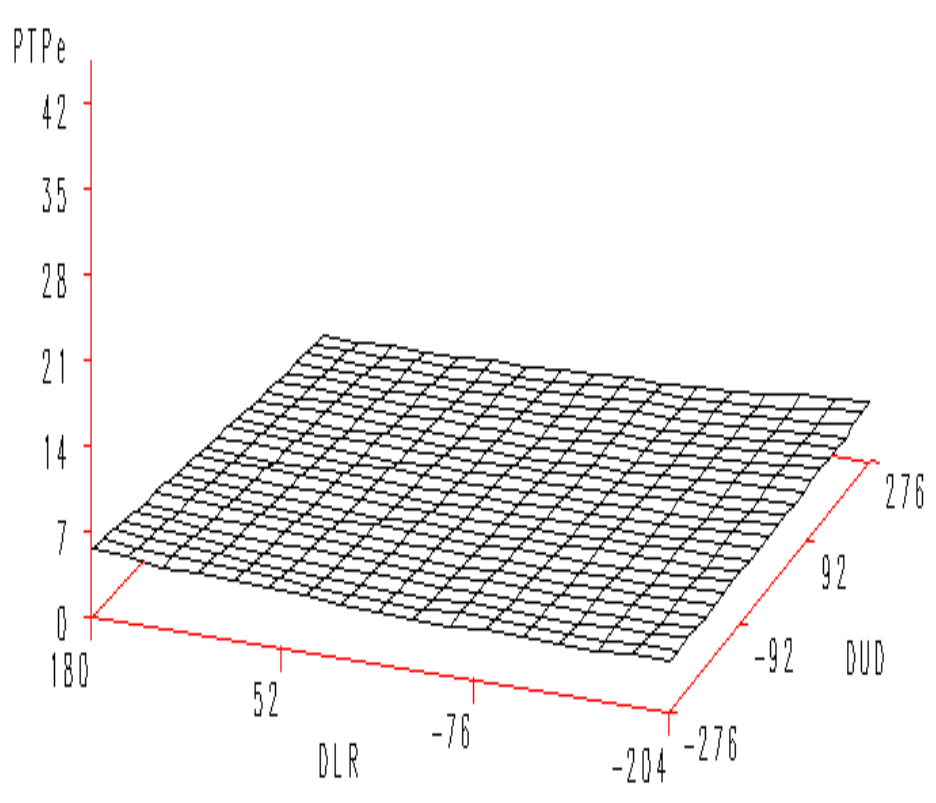

$D L R=D$ istance $(\mathrm{ft})$ Left to Riqht, DUD $=$ Distance $(\mathrm{ft}) \mathrm{UD}_{\mathrm{p}}$ to Down, Up $=$ North

Figure 2a. Fitted response surfaces of predicted total pests in fields with crimson clover as the cover crop. Surfaces shown are for sampling weeks 1 and 2. From data taken from cotton fields during the cover crop phase in Tift county, Georgia in 2001. 


\section{Cover Crop Effect on Numbers of Insects on Cotton}

Sumple Time=Cover Crop, Cover Crop=Crimson Clover

PTPe = Predicted Totol Pests

Sampletleek=3

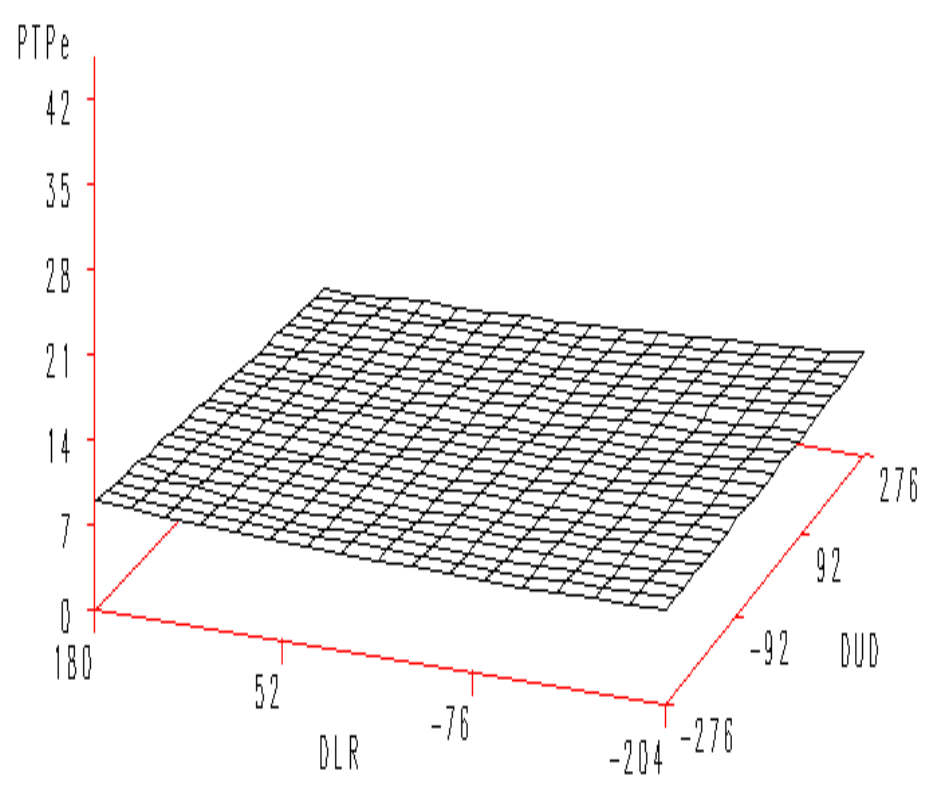

DLR = Distance $(\mathrm{ft})$ Left to Riqht, DUD = Distance $(\mathrm{ft})$ Up to Down, Up = North

\section{Cover Crop Effect on Numbers of Insects on Cotton}

Somple Time=Cover Crop, Cover Crop=Crimson Clover

PTPe = Predicted Totol Pests

Somple elleek=4

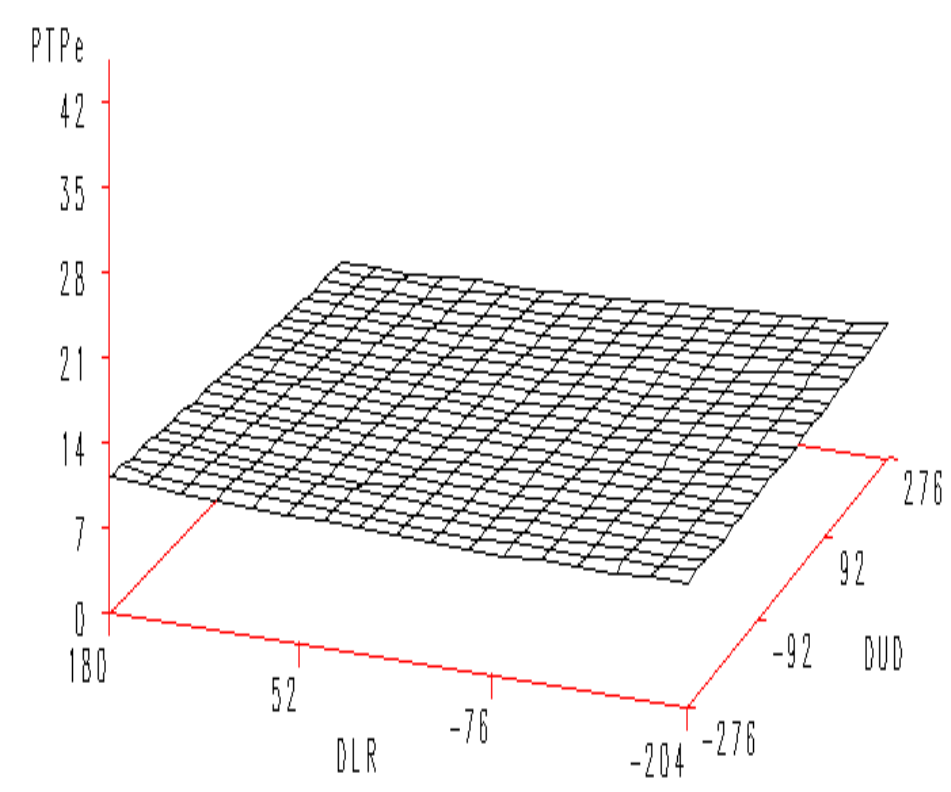

$D L R=D$ istance $(\mathrm{ft})$ Left to Riqht, DUD $=D$ istance $(\mathrm{ft})$ Up to Down, Up $=$ North

Figure 2b. Fitted response surfaces of predicted total pests in fields with crimson clover as the cover crop. Surfaces shown are for sampling weeks 3 and 4 . From data taken from cotton fields during the cover crop phase in Tift county, Georgia in 2001. 


\section{Cover Crop Effect on Numbers of Insects on Cotton}

SumpleTime=Cover Crop, Cover Crop=Crimson Clover

$$
\begin{aligned}
\text { PTPe }= & \text { Predicted Totol Pests } \\
\text { Sompletleek }=5 &
\end{aligned}
$$

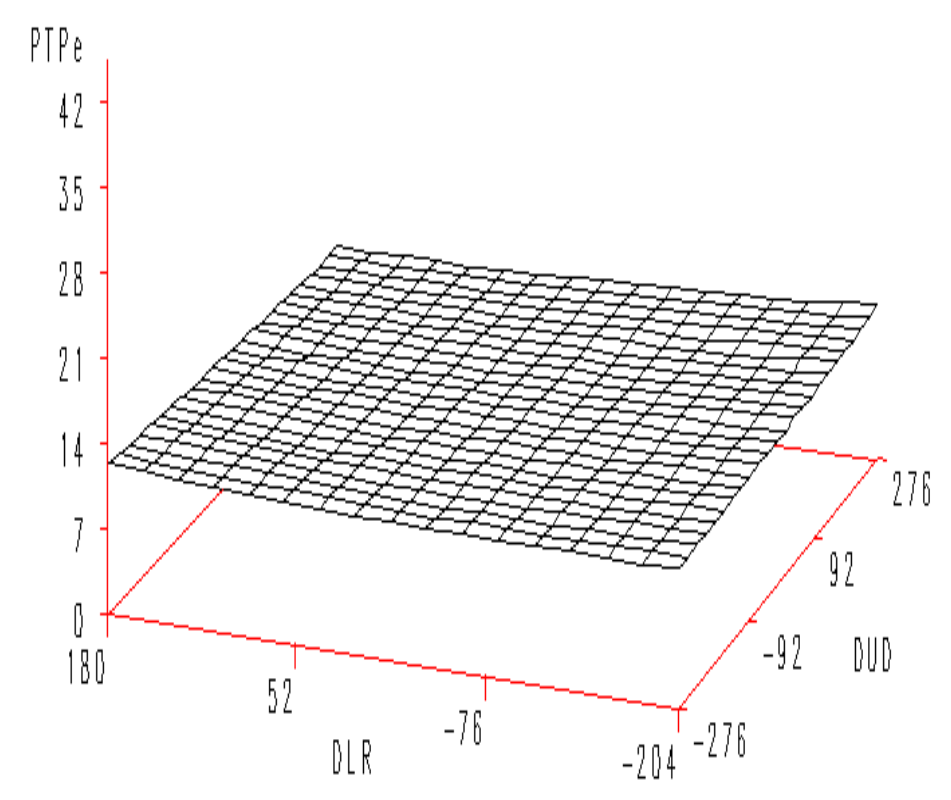

$D L R=$ Distance $(f t)$ Left to Riaht, DUD $=$ Distance $(f t) U_{p}$ to Down, Up $=$ North

\section{Cover Crop Effect on Numbers of Insects on Cotton \\ Somple Time=Cover Crop, Cover Crop=Crimson Clover \\ PTPe = Predicted Totol Pests \\ Somple elleek=6}

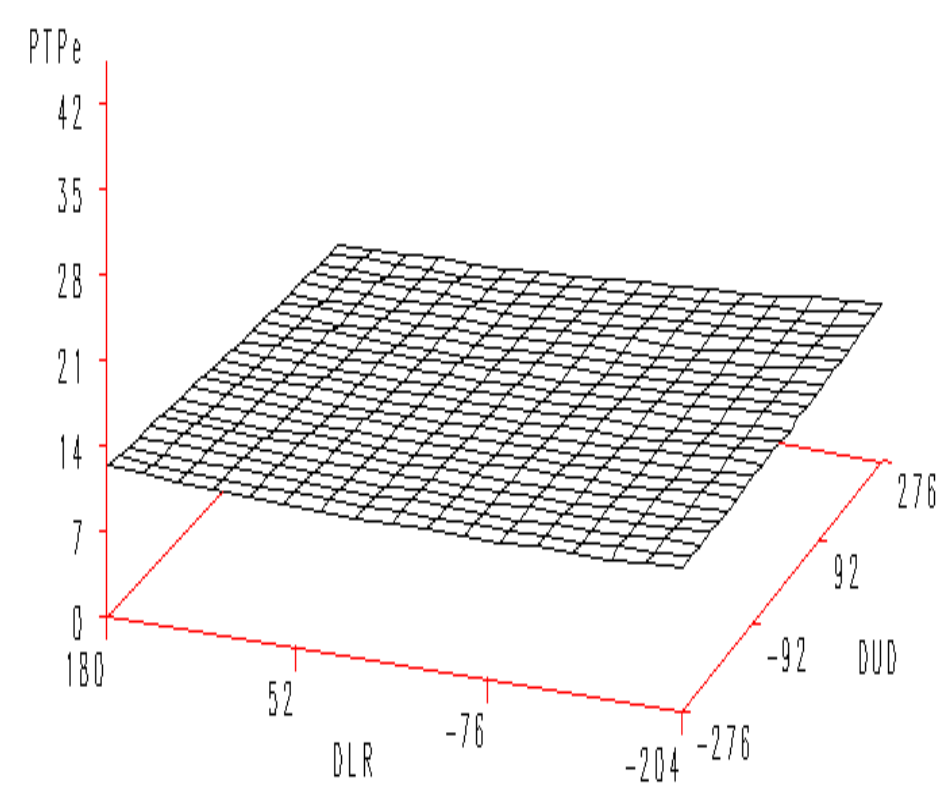

$D L R=D$ istance $(f t)$ Left to Riaht, DUD $=$ Distance $(f t) U_{D}$ to Down, $U_{D}=$ North

Figure 2c. Fitted response surfaces of predicted total pests in fields with crimson clover as the cover crop. Surfaces shown are for sampling weeks 5 and 6 . From data taken from cotton fields during the cover crop phase in Tift county, Georgia in 2001. 
Cover Crop Effect on Numbers of Insects on Cotton

Somple Time=Cover Crop, Cover Crop=Crimson Clover

PTPe = Predicted Totol Pests

Sompletleek=?

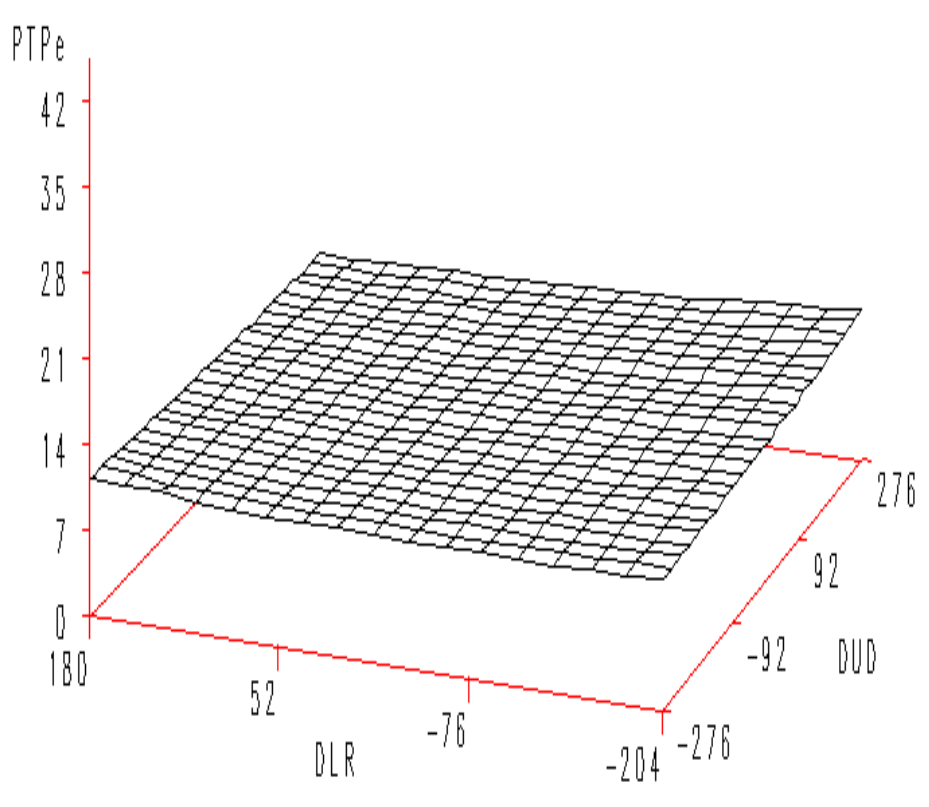

DLR = Distance ( $\mathrm{ft}$ ) Left to Riqht, DUD = Distance $(\mathrm{ft})$ Up to Doun, Up = North

\section{Cover Crop Effect on Numbers of Insects on Cotton}

Somple Time=Cover Crop, Cover Crop=Crimson Clover

$$
\text { PTPe }=\text { Predicted Totol Pests }
$$

Sompletleek=8

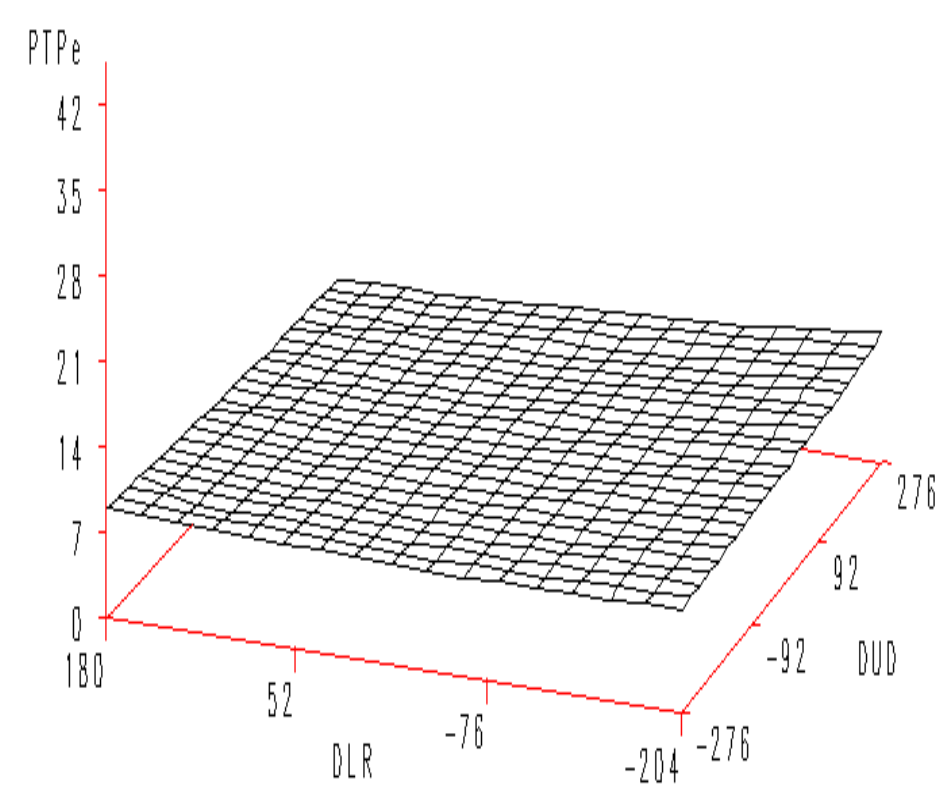

$D L R=D$ istance $(f t)$ Left to Right, DUD $=$ Distance $(\mathrm{ft})$ Up to Down, $U_{p}=$ North

Figure 2d. Fitted response surfaces of predicted total pests in fields with crimson clover as the cover crop. Surfaces shown are for sampling weeks 7 and 8 . From data taken from cotton fields during the cover crop phase in Tift county, Georgia in 2001. 


\section{Cover Crop Effect on Numbers of Insects on Cotton}

somple Time=Cover Crop, CoverCrop=Crimson Clover

PTPe = Predicted Totol Pests

Sompleteek $=9$

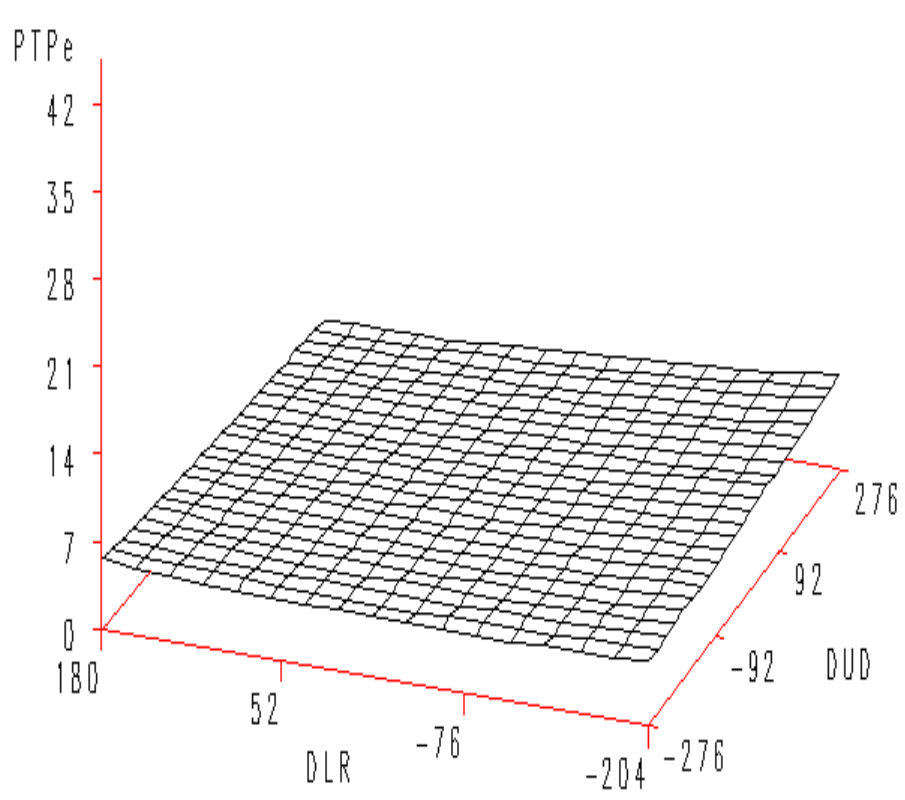

DLR = Distance $(f t)$ Left to Right, DUD = Distance $(f t)$ Up to Down, Up $=$ North

\section{Cover Crop Effect on Numbers of Insects on Cotton}

Sompletime=Cover Crop, CoverCrop=Crimson Clover

PTPe = Predicted Totol Pests

Samp letheek $=10$

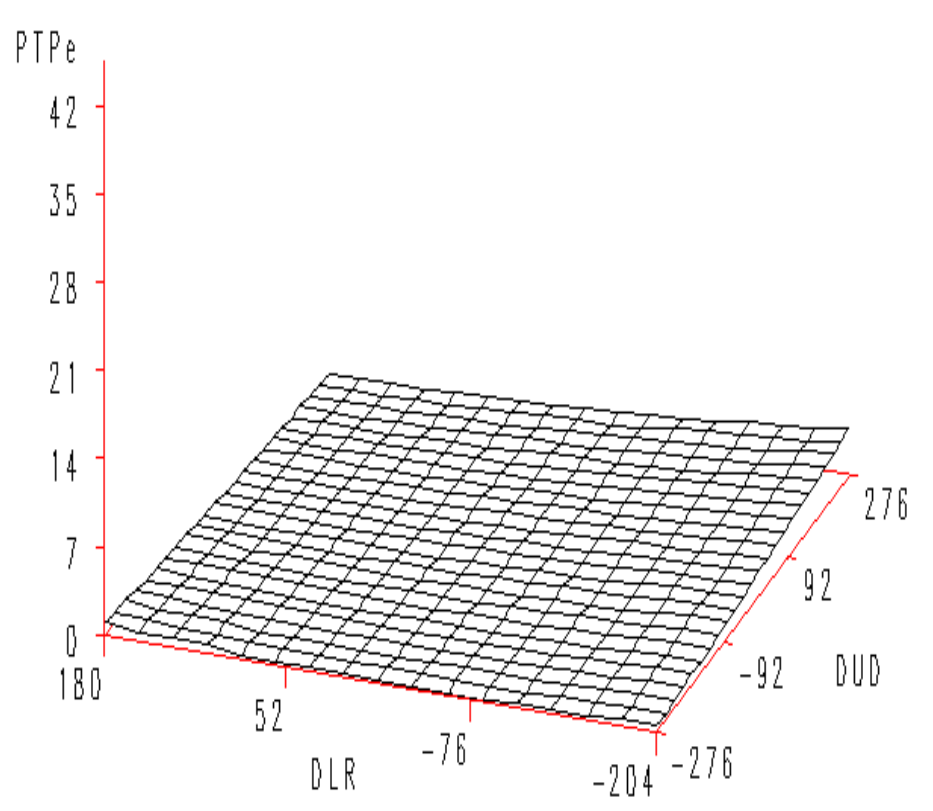

$D L R=D$ istance $(f t)$ Left to Right, DUD = Distance $(f t)$ Up to Down, Up $=$ North

Figure 2e. Fitted response surfaces of predicted total pests in fields with crimson clover as the cover crop. Surfaces shown are for sampling weeks 9 and 10. From data taken from cotton fields during the cover crop phase in Tift county,

Georgia in 2001. 


\section{Cover Crop Effect on Numbers of Insects on Cotton \\ Sampletime=Cotton, CoverCrop=Crimson Clover \\ PTPe = Predicted Totol Pests \\ Somp letheek=14}

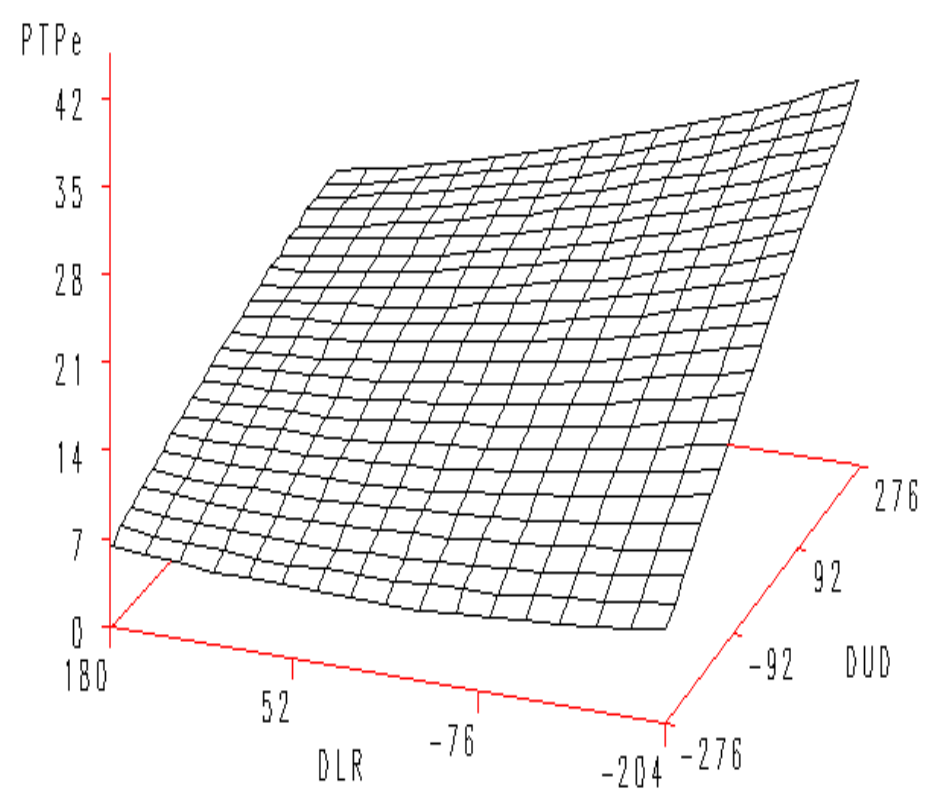

DLR $=$ Distance $(f t)$ Left to Right, DUD $=$ Distance $(f t)$ Up to Down, Up $=$ North

\section{Cover Crop Effect on Numbers of Insects on Cotton}

Sample Time= Cotton, Cover Crop=Crimson Clover

PTPe = Predicted Totol Pests

Sompletheek $=15$

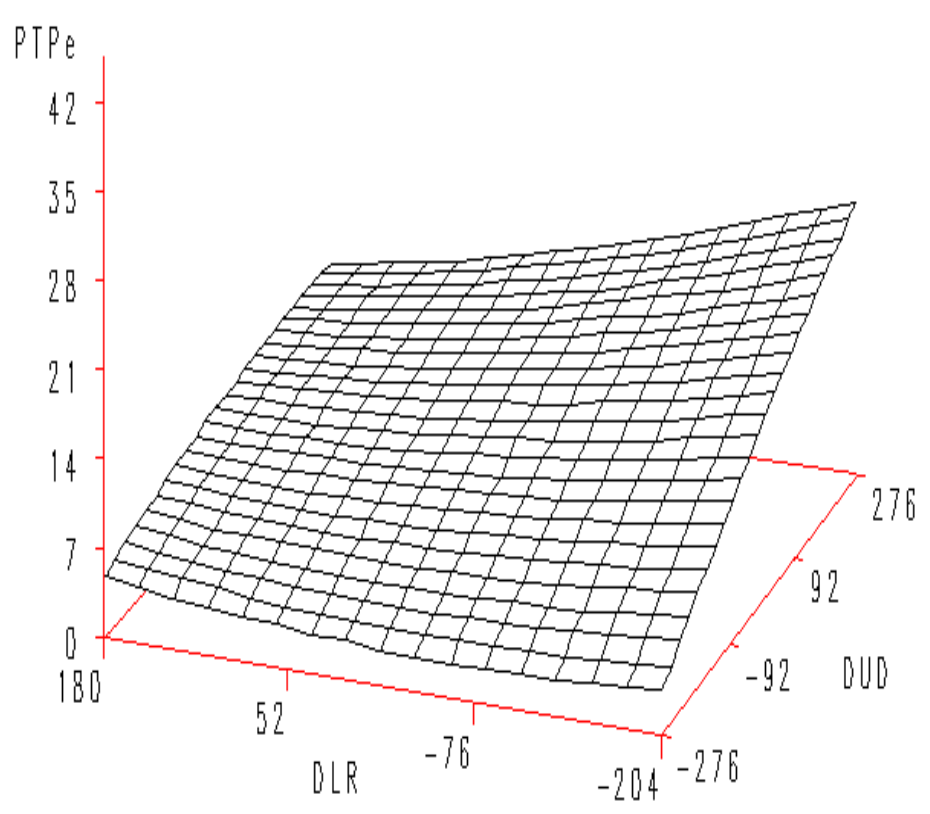

DLR $=$ Distance $(\mathrm{ft})$ Left to Right, DUD $=$ Distance $(\mathrm{ft})$ Up to Down, Up $=$ North

Figure 3a. Fitted response surfaces of predicted total pests in fields with crimson clover as the cover crop. Surfaces shown are for sampling weeks 14 and 15. From data taken from cotton fields during the cotton crop phase in Tift county, Georgia in 2001 


\section{Cover Crop Effect on Numbers of Insects on Cotton \\ sampletime=Cotton, CoverCrod=Crimson Clover \\ PTPe = Predicted Totol Pests \\ Sompletheek=16}

\section{Cover Crop Effect on Numbers of Insects on Cotton \\ Sompletime=Cotton, CoverCrop=Crimson Clover \\ PTPe = Predicted Totol Pests Sump leteek $=17$}

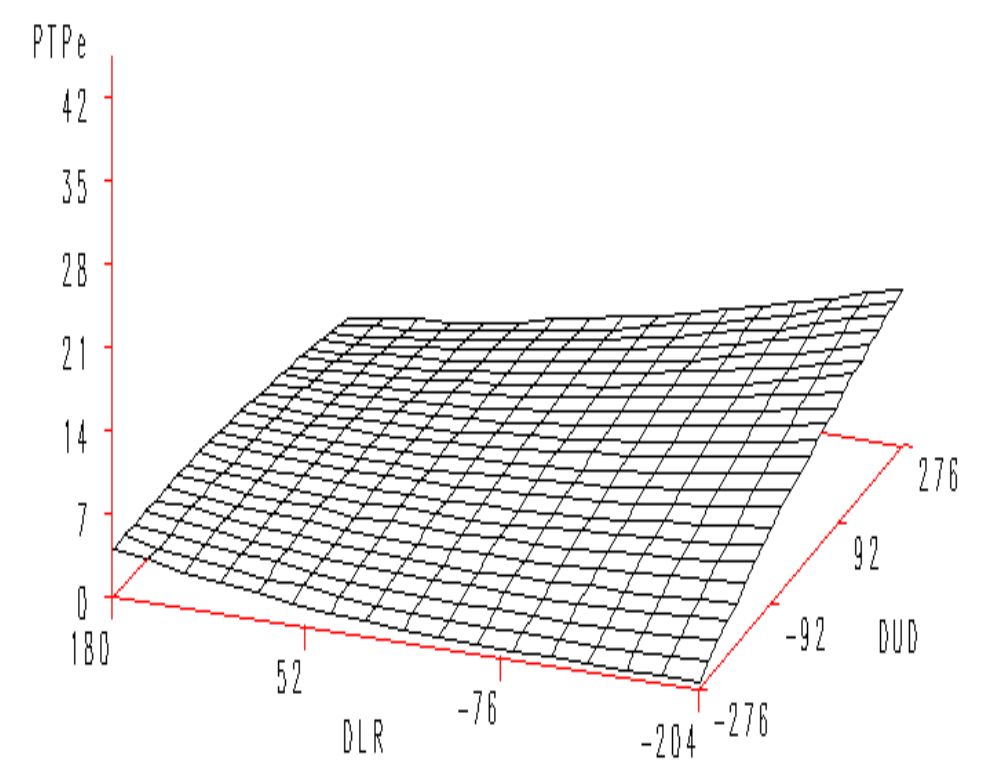

$D L R=$ Distance $(\mathrm{ft})$ Left to Riqht, DUD $=$ Distance $(\mathrm{ft}) \mathrm{Up}_{\mathrm{p}}$ to Down, $\mathrm{Up}_{\mathrm{p}}=$ North

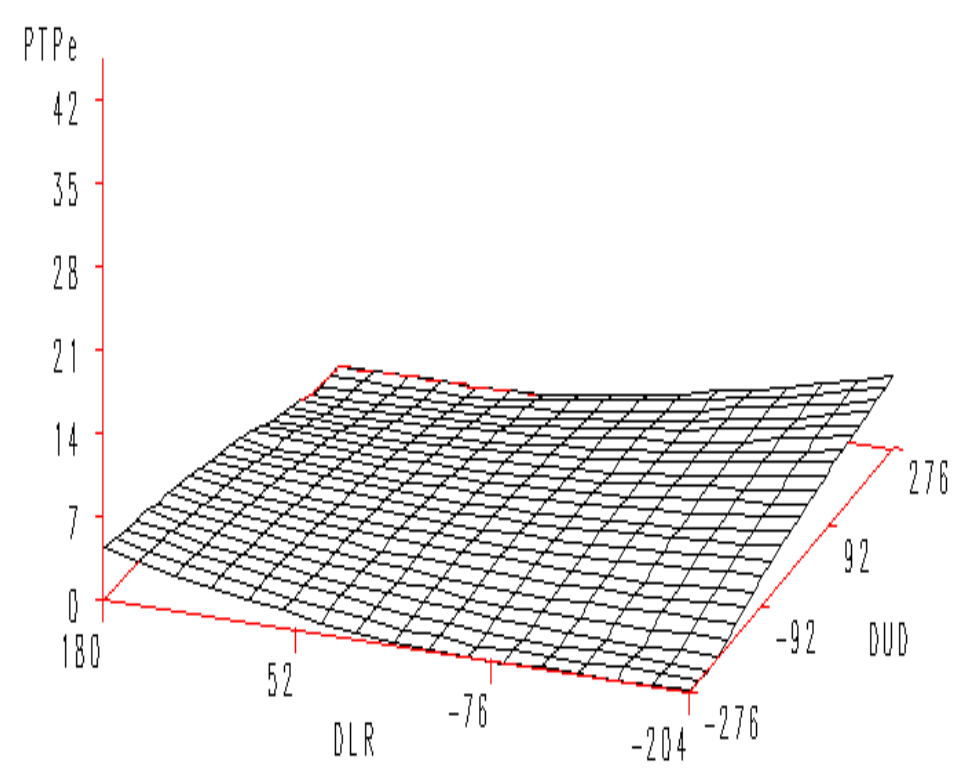

$D L R=$ Distance $(f t)$ Left to Right, DUD $=$ Distance $(f t)$ Up to Down, $U_{p}=$ North

Figure 3b. Fitted response surfaces of predicted total pests in fields with crimson clover as the cover crop. Surfaces shown are for sampling weeks 16 and 17. From data taken from cotton fields during the cotton crop phase in Tift county, Georgia in 2001. 
Cover Crop Effect on Numbers of Insects on Cotton

Sampletime=Cot ton, CoverCrop=Crimson Clover

PTPe = Predicted Totol Pests

Sumpletleek=18

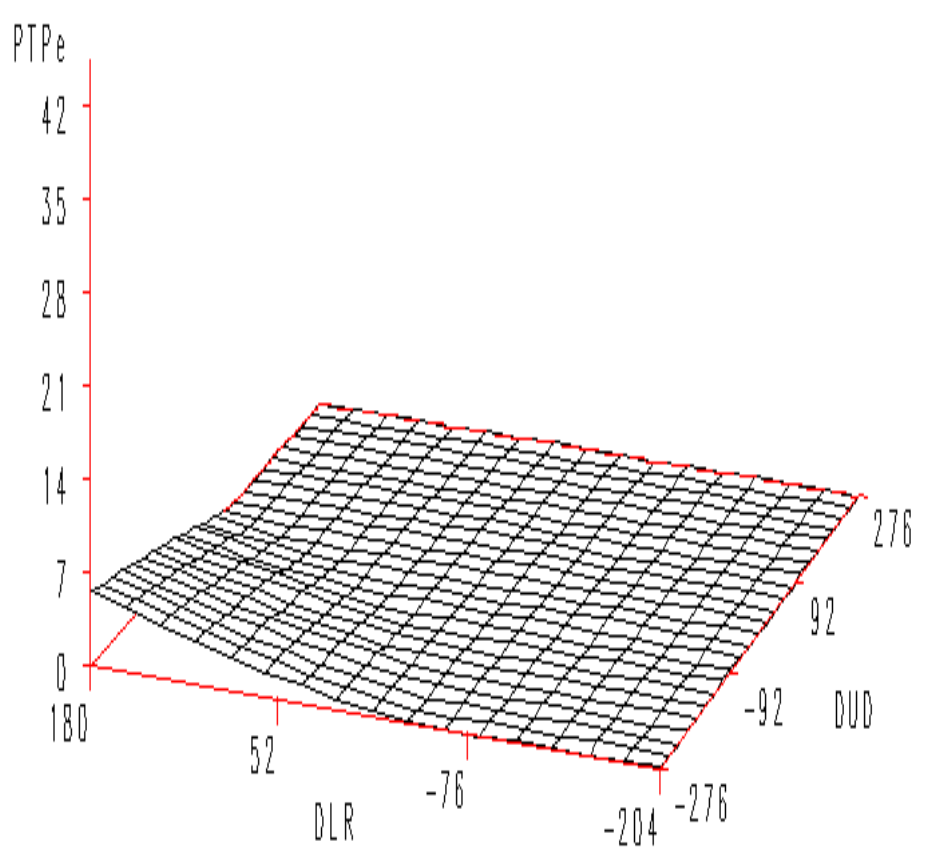

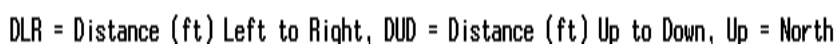

Figure 3c. Fitted response surface of predicted total pests in fields with crimson clover as the cover crop. Surface shown is for sampling week 18 . From data taken from cotton fields during the cotton crop phase in Tift county, Georgia in 2001. 


\section{Cover Crop Effect on Numbers of Insects on Cotton \\ Sumpletime=Cover Crop, Cover Crop=Crimson Clover PTPr = Predicted Totol Predotors \\ Sompletheek $=1$}

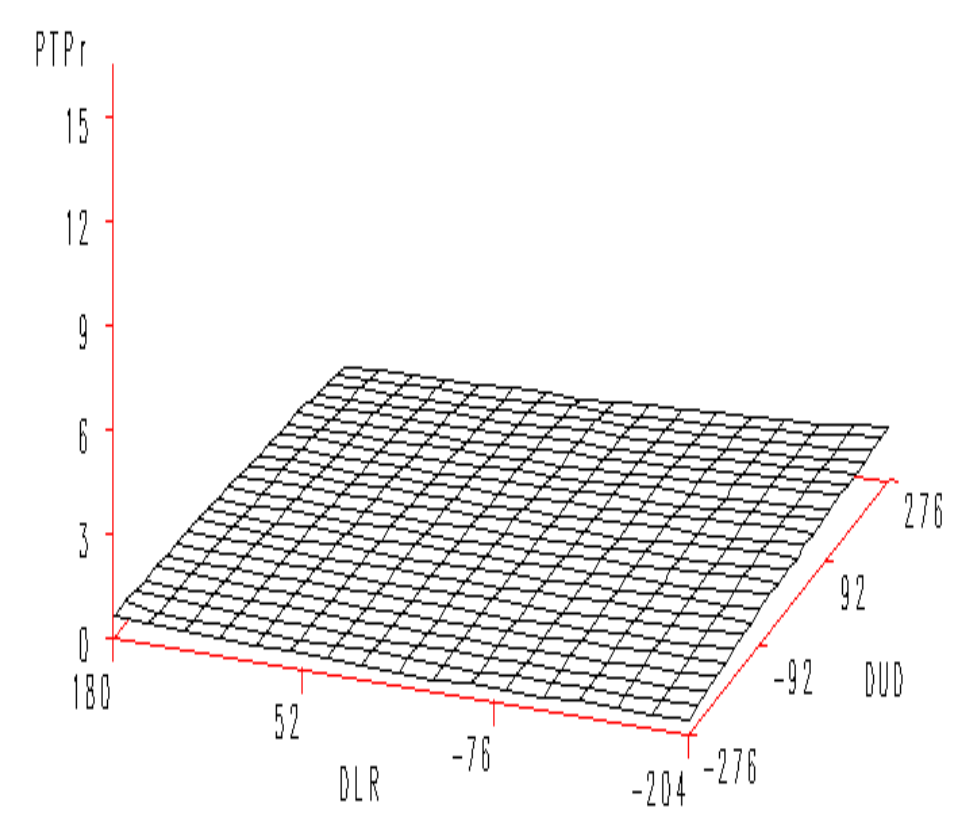

DLR = Distance $(\mathrm{ft})$ Left to Riaht, DUD $=$ Distance $(\mathrm{ft}) U_{\mathrm{p}}$ to Down, $U_{\mathrm{p}}=$ North

\section{Cover Crop Effect on Numbers of Insects on Cotton \\ Sampletime=Cover Crop, Cover Crop=Crimson Clover \\ PTPr = Predicted Totol Predotors \\ Sompletheek $=2$}

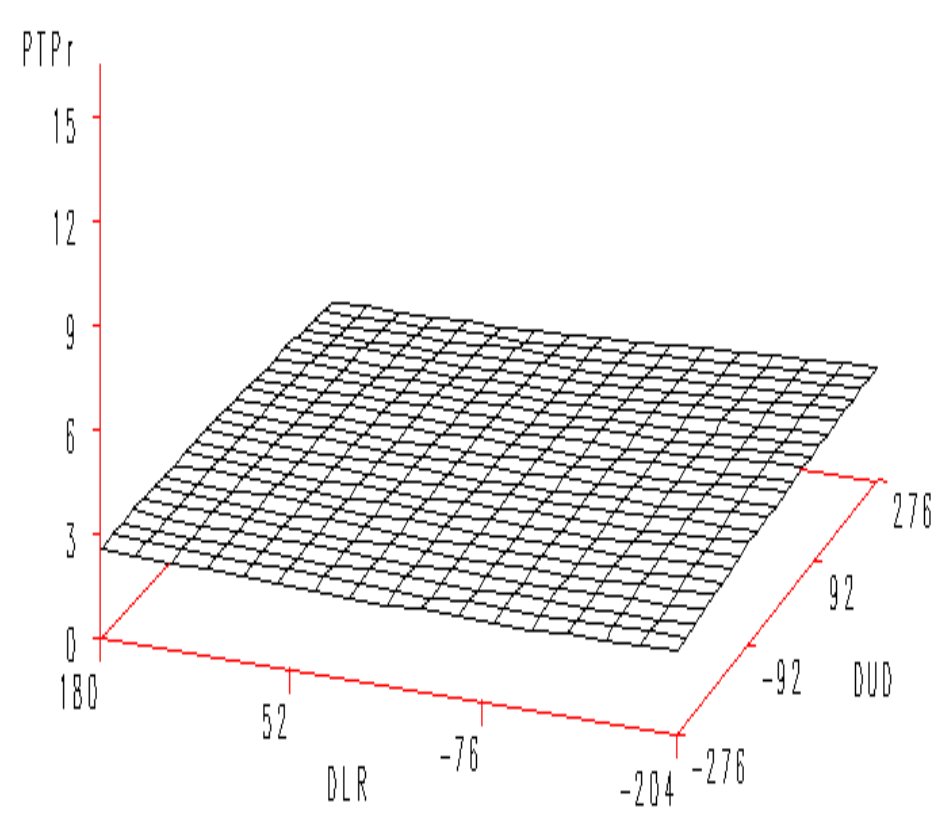

$D L R=D i s t a n c e(f t)$ Left to Right, DUD $=$ Distance $(f t) U_{D}$ to Down, Up = North

Figure 4a. Fitted response surfaces of predicted total predators in fields with crimson clover as the cover crop. Surfaces shown are for sampling weeks 1 and 2. From data taken from cotton fields during the cover crop phase in Tift county, Georgia in 2001. 


\section{Cover Crop Effect on Numbers of Insects on Cotton \\ Sample Time =Cover Crop, CoverCrop=Crimson Clover PTPr = Predicted Totol Predators Sompletheek=3}

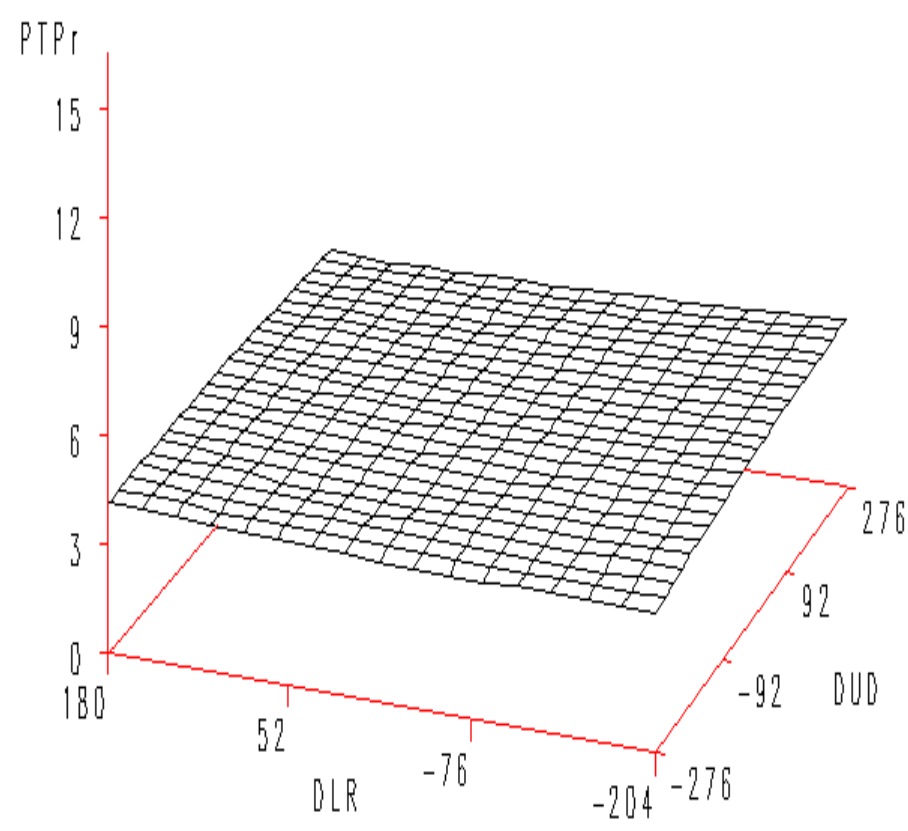

DLR $=$ Distance $(f t)$ Left to Right, DUD $=$ Distance $(f t)$ Up to Down, Up $=$ North

\section{Cover Crop Effect on Numbers of Insects on Cotton}

Sumpletime=Cover Crop, Cover Crop=Crimson Clover

PTPr = Predicted Totol Predutors

Sompletheek=4

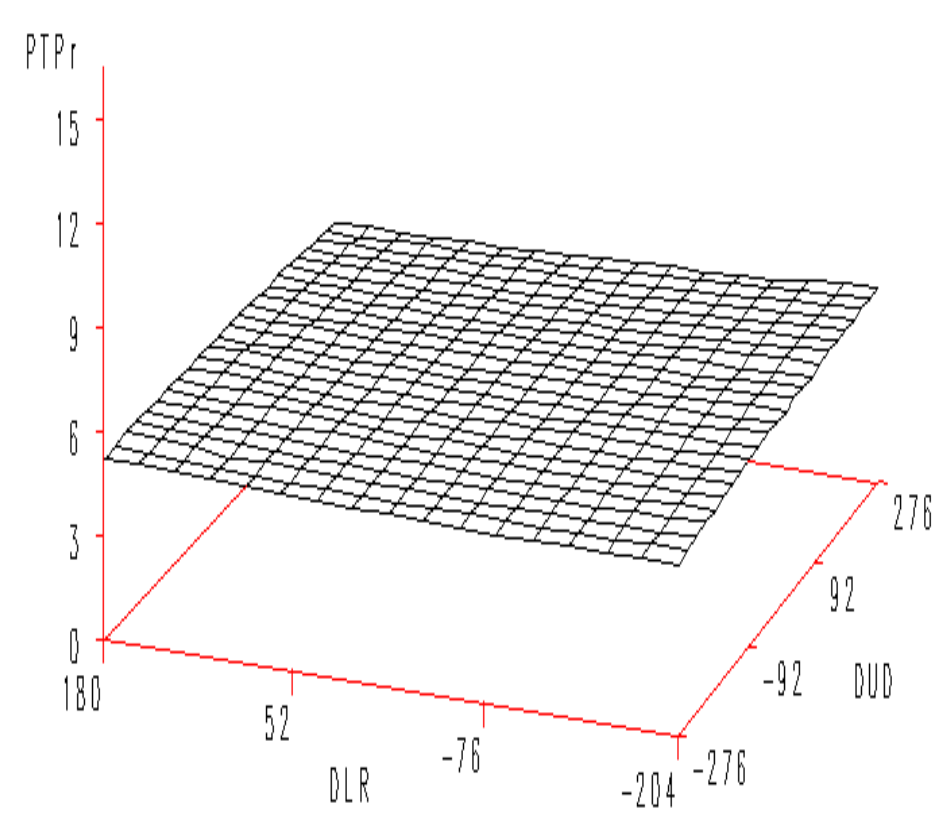

$D L R=D i s t a n c e(f t)$ Left to Right, DUD $=$ Distance $(f t)$ Up to Down, Up = North

Figure 4b. Fitted response surfaces of predicted total predators in fields with crimson clover as the cover crop. Surfaces shown are for sampling weeks 3 and 4 . From data taken from cotton fields during the cover crop phase in Tift county, Georgia in 2001. 


\section{Cover Crop Effect on Numbers of Insects on Cotton}

Sample Time=Cover Crop, Cover Crop=Crimson Clover PTPr = Predicted Totol Predutors Sompletheek =?

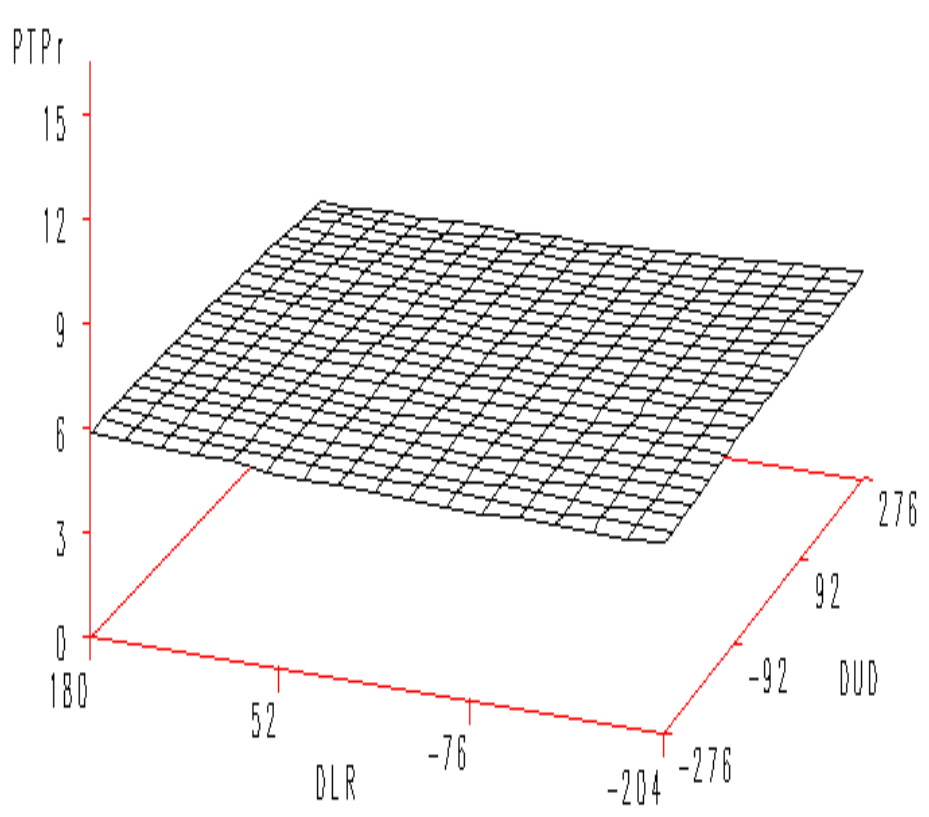

$D L R=D$ istance $(f t)$ Left to Right, DUD $=$ Distance $(f t) U_{p}$ to Down, $U_{p}=$ North

\section{Cover Crop Effect on Numbers of Insects on Cotton}

\author{
Sompletime=Cover Crop, CoverCrop=Crimson Clover \\ PTPr = Predicted Totol Predutors \\ Somp lelleek=8
}

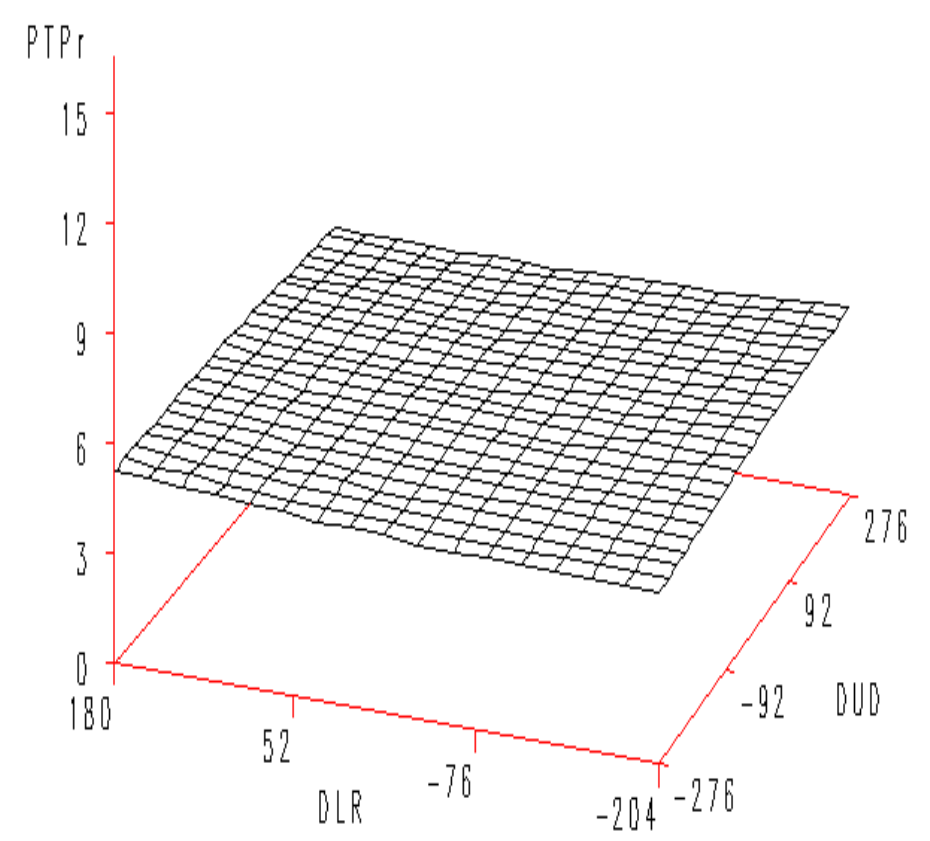

$D L R=$ Distance $(f t)$ Left to Riqht, DUD $=$ Distance $(\mathrm{ft})$ Up to Down, Up $=$ North Figure 4c. Fitted response surfaces

of predicted total predators in fields with crimson clover as the cover crop. Surfaces shown are for sampling weeks 5 and 6.

From data taken from cotton fields during the cover crop phase in Tift county, Georgia in 2001.

Figure 4d. Fitted response surfaces of predicted total predators in fields with crimson clover as the cover crop. Surfaces shown 


\section{Cover Crop Effect on Numbers of Insects on Cotton}

Sample Time=Cover Crop, Cover Crop=Crimson Clover PTPr = Predicted Totol Predutors Sompl elleek $=9$

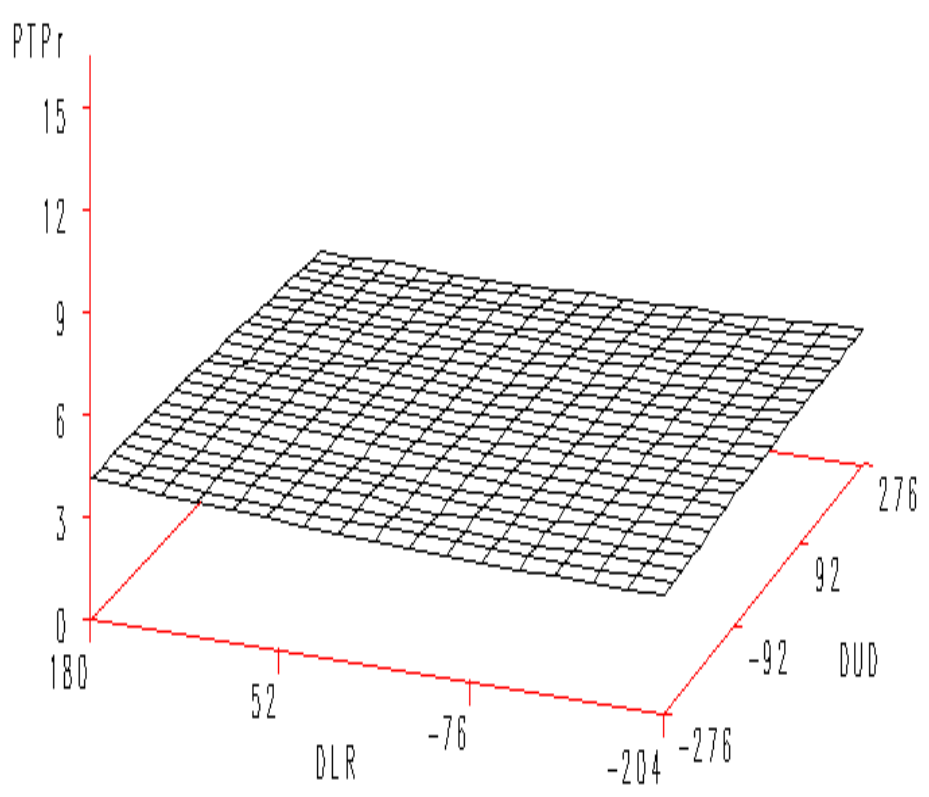

$D L R=D$ istance $(\mathrm{ft})$ Left to Right, DUD $=$ Distance $(\mathrm{ft})$ Up to Down, Up = North

\section{Cover Crop Effect on Numbers of Insects on Cotton}

Somple Time=Cover Crop, Cover Crop=Crimson Clover

PTPr = Predicted Totol Predators

Sompleteek $=10$

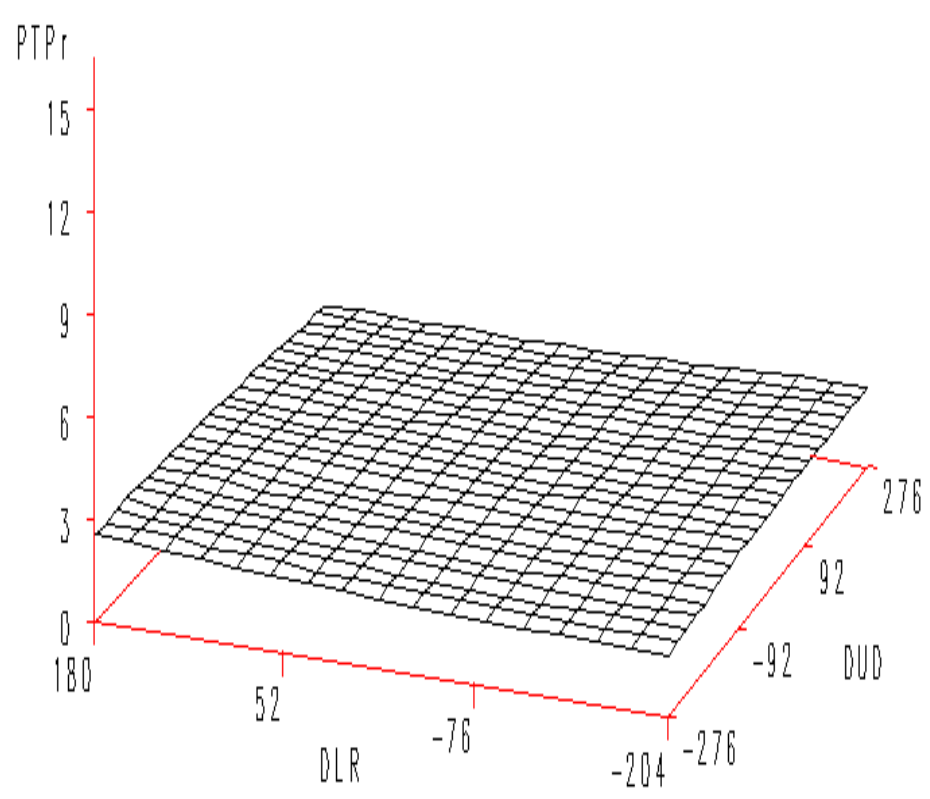

DLR = Distance $(\mathrm{ft})$ Left to Right, DUD $=$ Distance $(\mathrm{ft})$ Up to Down, Up = North

Figure 4e. Fitted response surfaces of predicted total predators in fields with crimson clover as the cover crop. Surfaces shown are for sampling weeks 9 and 10. From data taken from cotton fields during the cover crop phase in Tift county, Georgia in 2001. 


\section{Cover Crop Effect on Numbers of Insects on Cotton}

Sampletime=Cotton, CoverCrop=Crimson Clover

$$
\text { PTPr = Predicted Total Predutors }
$$

Sompleteek $=14$

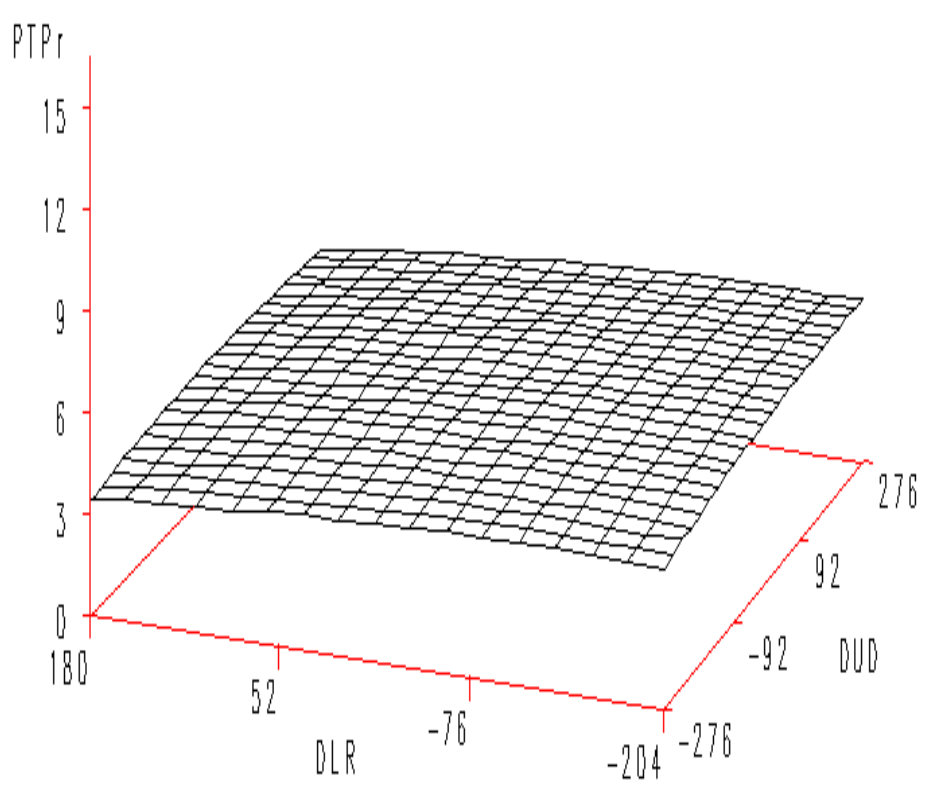

$D L R=D$ istance $(f t)$ Left to Riqht, DUD $=$ Distance $(f t)$ Up to Down, $U p=$ North

\section{Cover Crop Effect on Numbers of Insects on Cotton}

SumpleTime=Cotton, CoverCrop=Crimson Cloyer

$$
\text { PTPr = Predicted Totol Predutors }
$$

Sumpletleek $=15$

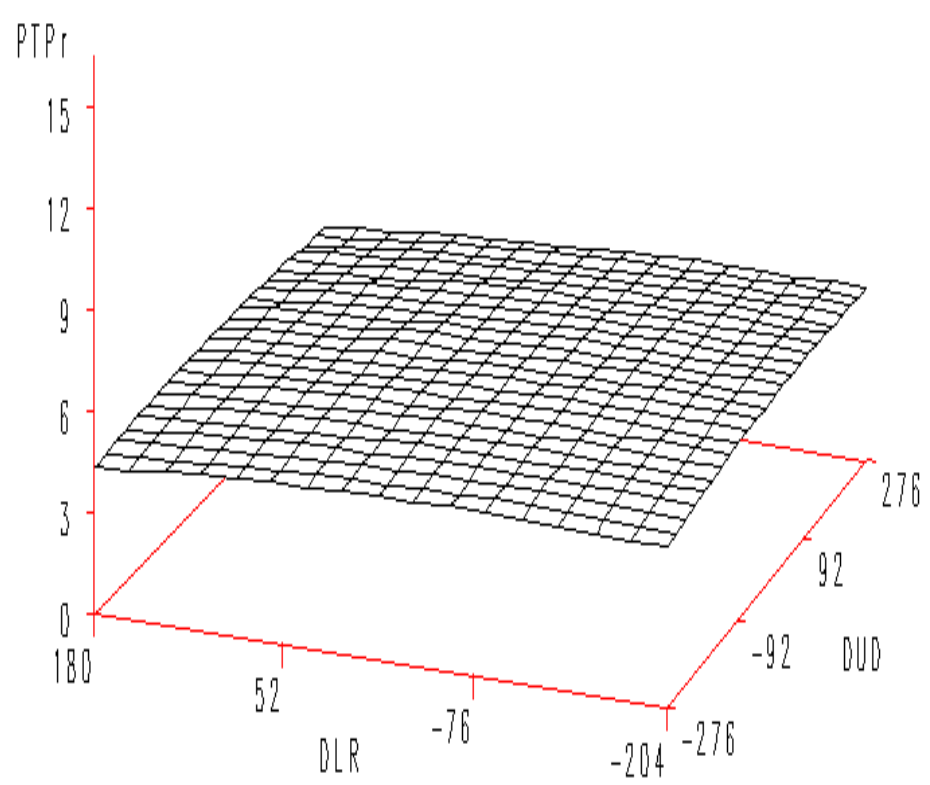

$D L R=D$ istance $(\mathrm{ft})$ Left to Riqht, DUD $=$ Distance $(\mathrm{ft}) \mathrm{Up}_{\mathrm{p}}$ to Down, Up $=$ North

Figure 5a. Fitted response surfaces of predicted total predators in fields with crimson clover as the cover crop. Surfaces shown are for sampling weeks 14 and 15. From data taken from cotton fields during the cotton crop phase in Tift county, Georgia in 2001. 
Cover Crop Effect on Numbers of Insects on Cotton

Sampletime=Cotton, CoverCrop=Crimson Clover PTPr = Predicted Totol Predutors Sump lethe $=16$

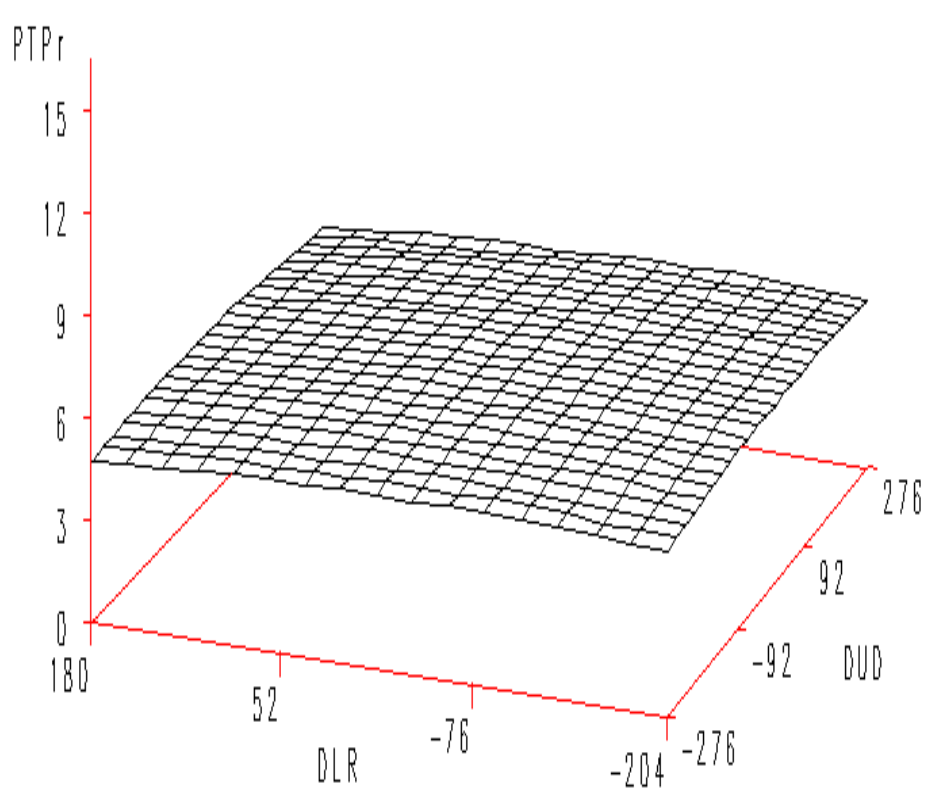

$D L R=D$ istance $(\mathrm{ft})$ Left to Right, DUD $=$ Distance $(\mathrm{ft})$ Up to Down, Up $=$ North

\section{Cover Crop Effect on Numbers of Insects on Cotton \\ Sumple Time=Cotton, CoverCrop=Crimson Clover \\ PTPr = Predicted Totol Predators \\ Somp lewe ek $=17$}

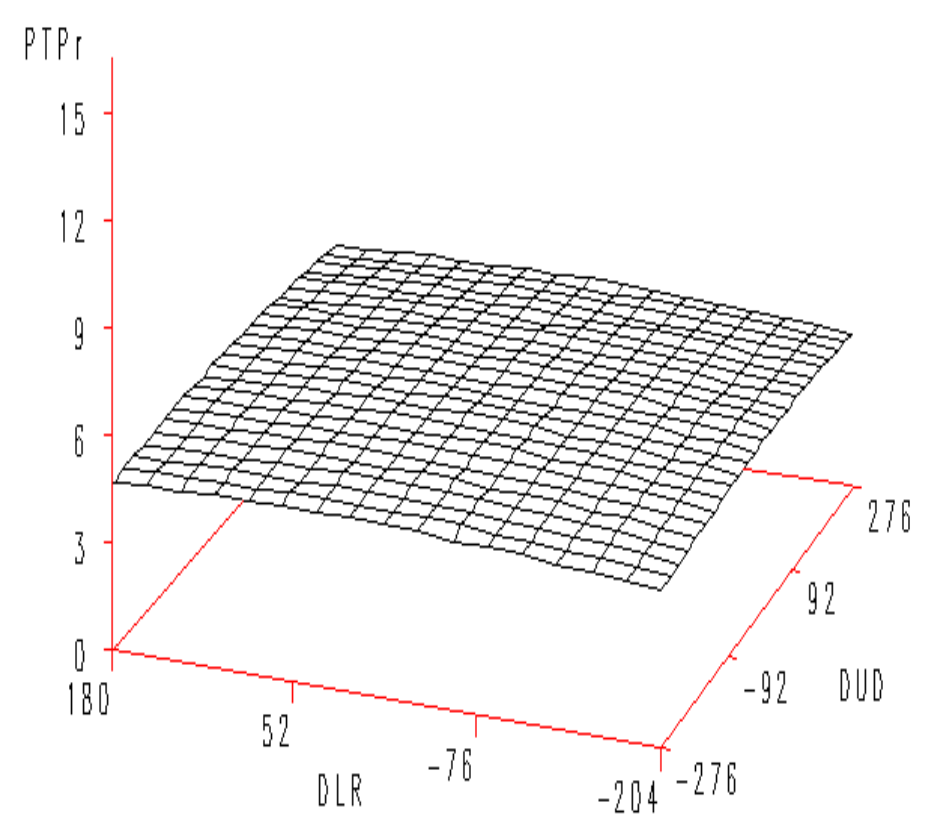

DLR = Distance $(\mathrm{ft})$ Left to Riqht, DUD $=$ Distance $(\mathrm{ft})$ Up to Down, Up $=$ North

Figure 5b. Fitted response surfaces of predicted total predators in fields with crimson clover as the cover crop. Surfaces shown are for sampling weeks 16 and 17. From data taken from cotton fields during the cotton crop phase in Tift county, Georgia in 2001. 


\section{Cover Crop Effect on Numbers of Insects on Cotton \\ Sompletime=Cotton, CoverCrop=Crimson Clover PTPT = Predicted Totol Predutors Sompleteek=18}

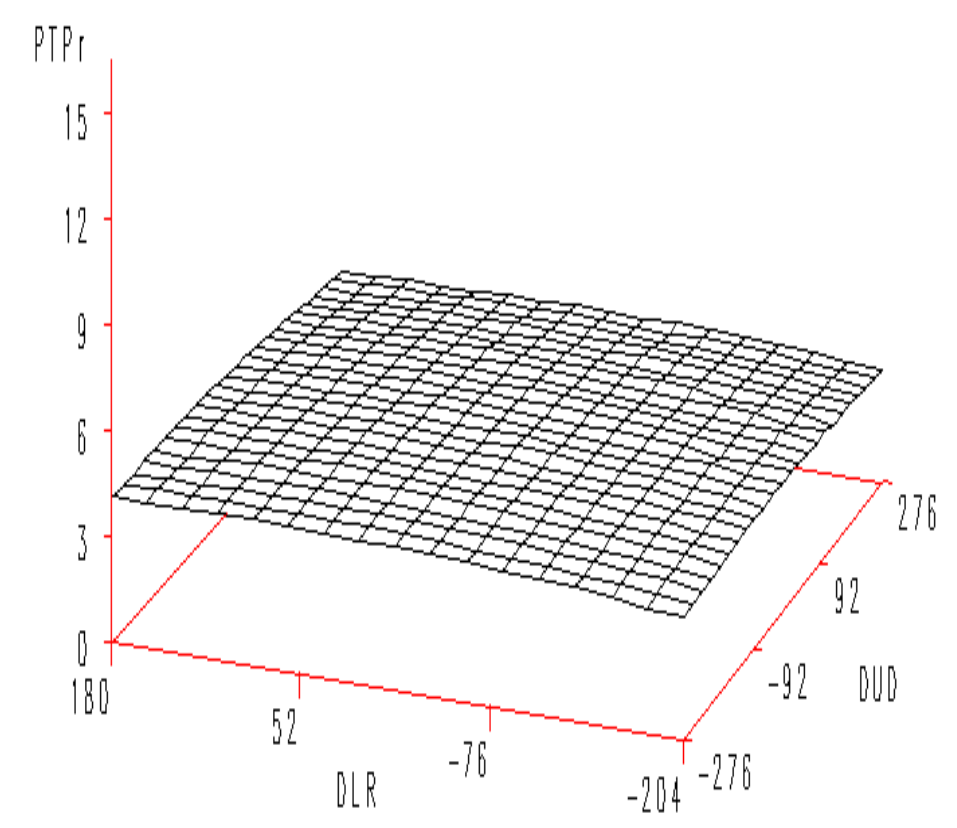

$D L R=D$ istance $(f t)$ Left to Riqht, DUD = Distance $(f t) U_{p}$ to Down, Up = North
Figure 5c. Fitted response surface of predicted total predators in fields with crimson clover as the cover crop. Surface shown is for sampling week 18. From data taken from cotton fields during the cotton crop phase in Tift county, Georgia in 2001. 
Cover Crop Effect on Numbers of Insects in Cotton

Sumple Time=Cover Crop, Cover Crop=Crimson Cloyer PTPo = Predicted Totol Porositoids Sompl etheek =1

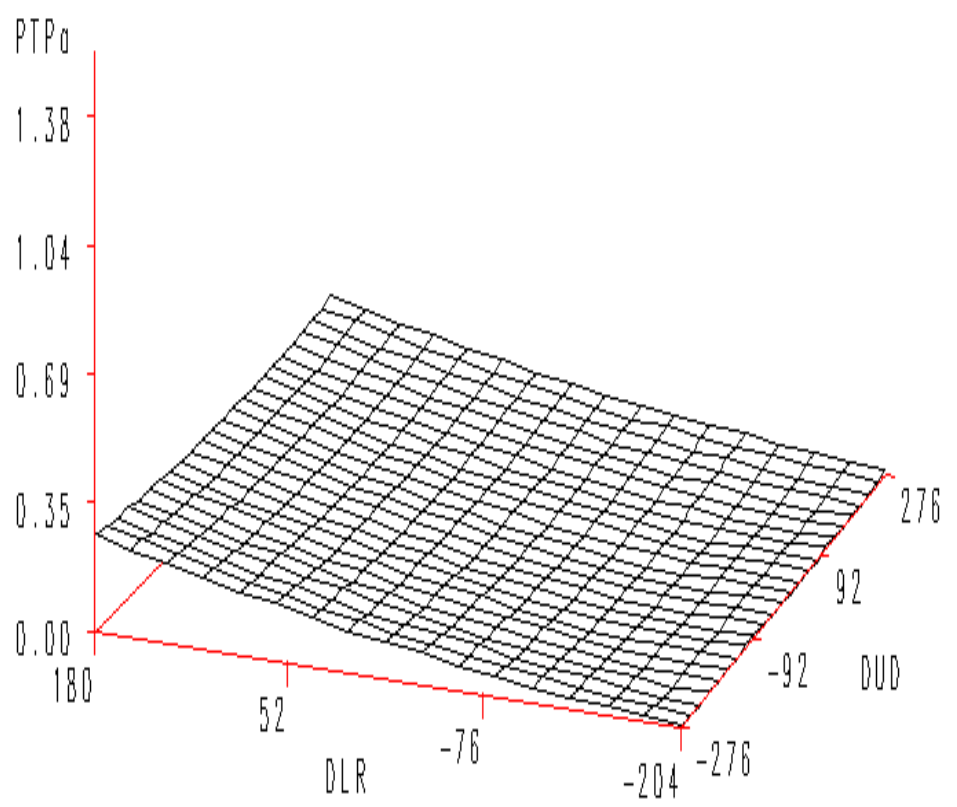

DI R = Dist.ance (ft.) I eft. t.n Rinht. DIIn = Dist.ann.e (ft.) Iln t.n Dnwn. Iln = Nnrth

\section{Cover Crop Effect on Numbers of Insects in Cotton}

Sampletime=Coyer Crop, CoverCrop=Crimson Clover PTPO = Predicted Total Porositoids Sompletherk=2

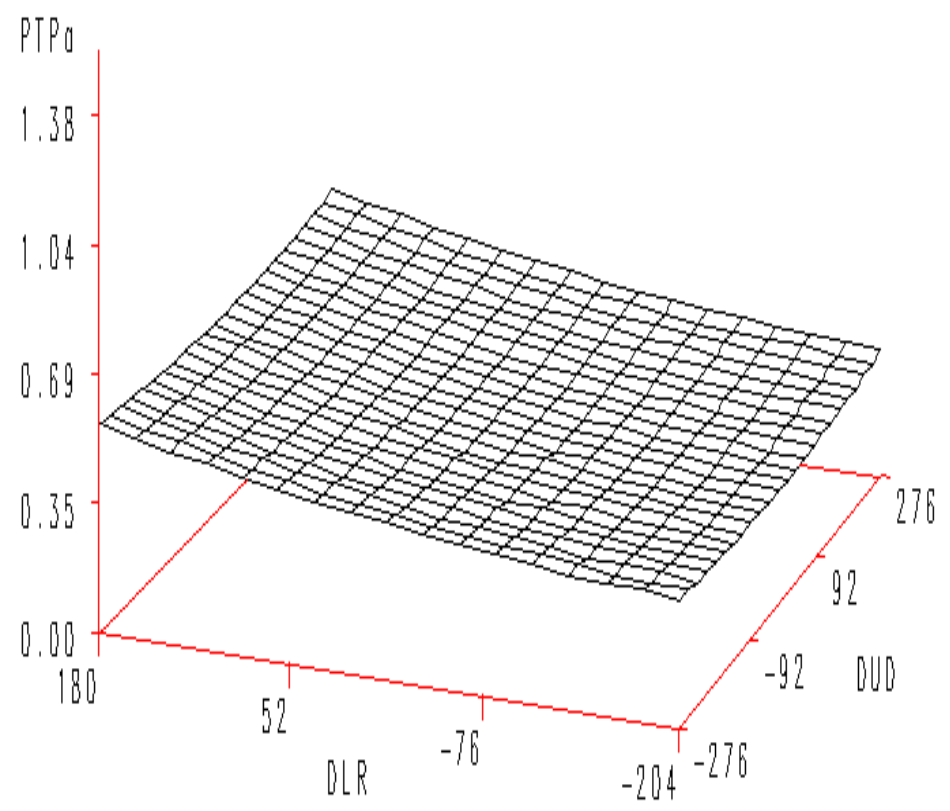

$D L R=$ Distance $(f t)$ Left to Right, DUD $=$ Distance $(f t)$ Up to Down, Up = North

Figure 6a. Fitted response surfaces of predicted total parasitoids in fields with crimson clover as the cover crop. Surfaces shown are for sampling weeks 1 and 2. From data taken from cotton fields during the cover crop phase in Tift county, Georgia in 2001. 


\section{Cover Crop Effect on Numbers of Insects on Cotton}

Sample Time=Cover Crop, CoverCrop=Crimson Clover

PTPu = Predicted Totol Porositoids

Sumpletheek=3

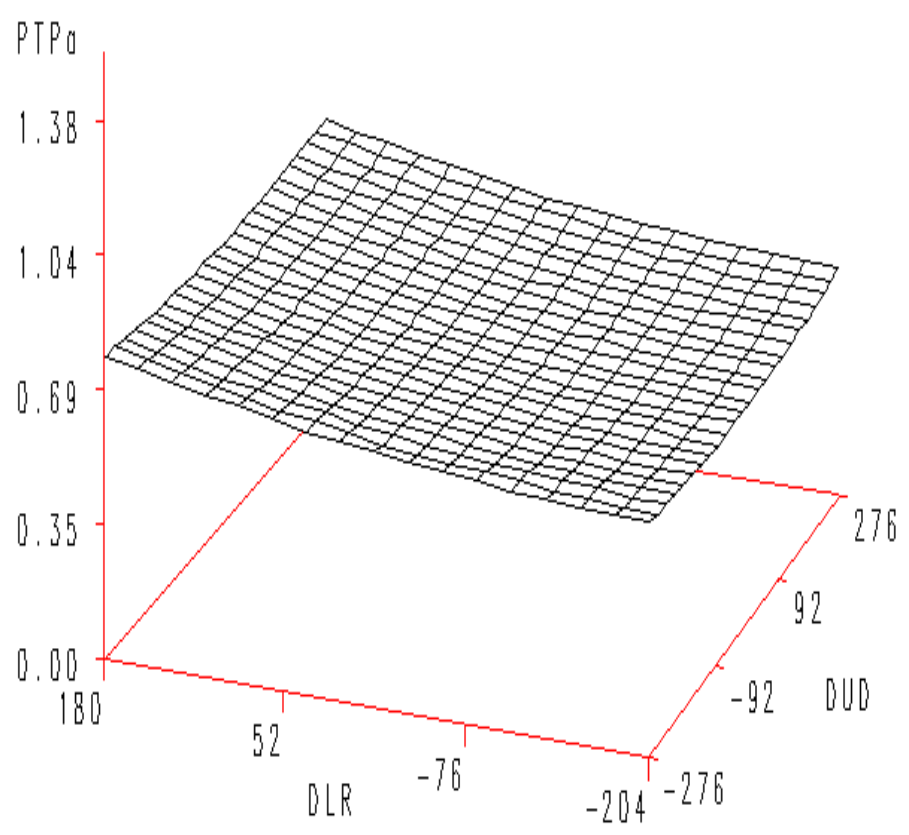

$D L R=$ Distance $(f t)$ Left to Right, DUD $=$ Distance $(f t)$ Up to Down, Up = North

\section{Cover Crop Effect on Numbers of Insects in Cotton}

Sompletime=Cover Crop, Cover Crop=Crimson Clover PTPu = Predicted Total Porasitoids Sompletleek $=4$

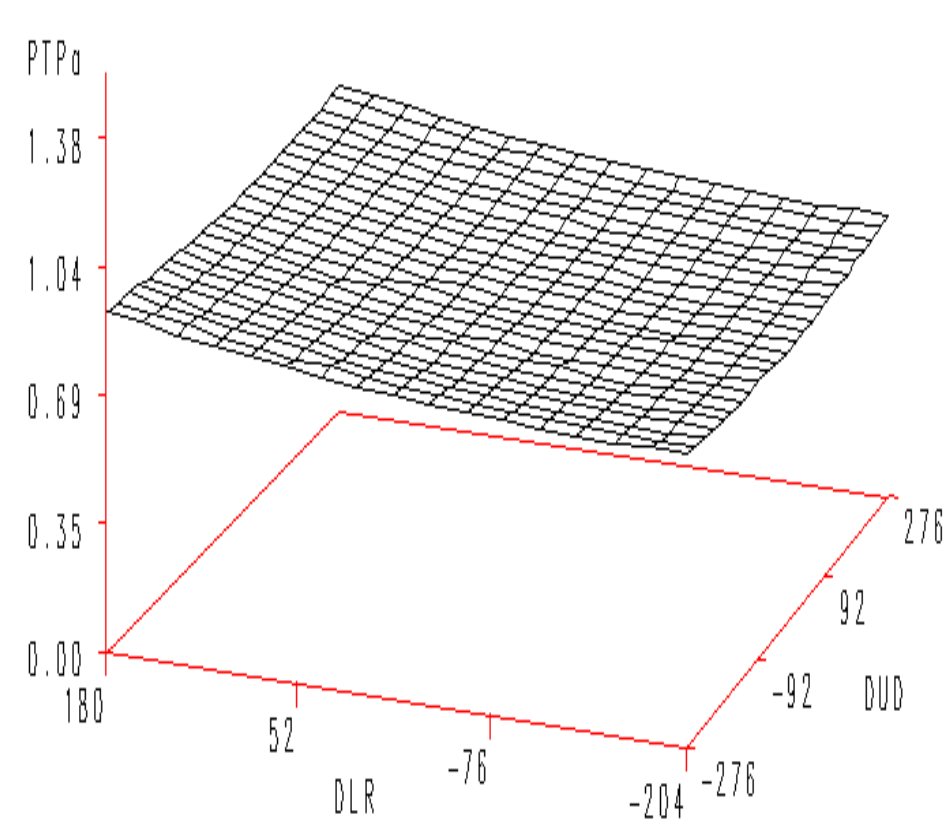

$D L R=D i s t a n c e(f t)$ Left to Right, DUD $=$ Distance $(f t) U_{D}$ to Down, Up $=$ North

Figure $6 \mathrm{~b}$. Fitted response surfaces of predicted total parasitoids in fields with crimson clover as the cover crop. Surfaces shown are for sampling weeks 3 and 4 . From data taken from cotton fields during the cover crop phase in Tift county, Georgia in 2001. 
Cover Crop Effect on Numbers of Insects in Cotton

Sompletime=Cover Crop, Cover Crop=Crimson Clover PTPo = Predicted Totol Porasitoids Sompl elleek=5

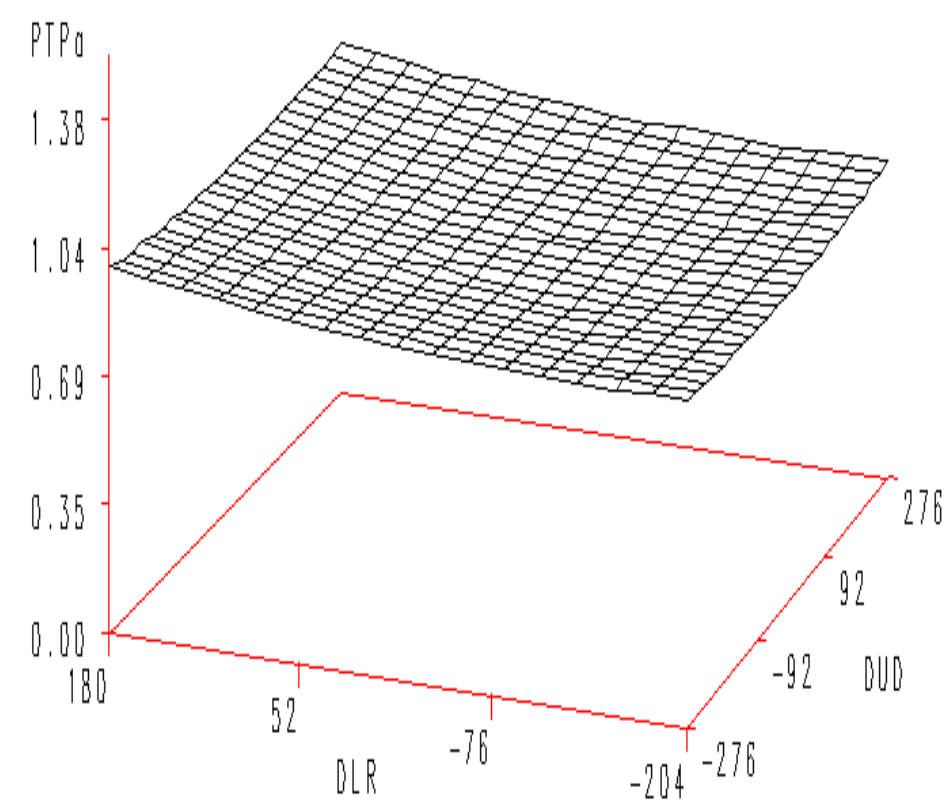

DLR = Distance $(\mathrm{ft})$ Left to Right, DUD $=$ Distance $(\mathrm{ft}) \mathrm{Up}_{\mathrm{p}}$ to Down, $\mathrm{Up}_{\mathrm{p}}=$ North

\section{Cover Crop Effect on Numbers of Insects in Cotton}

Sompletime=Coyer Crop, CoverCrop=Crimson Clover

PTPO = Predicted Total Porositoids

Sampletheek=6

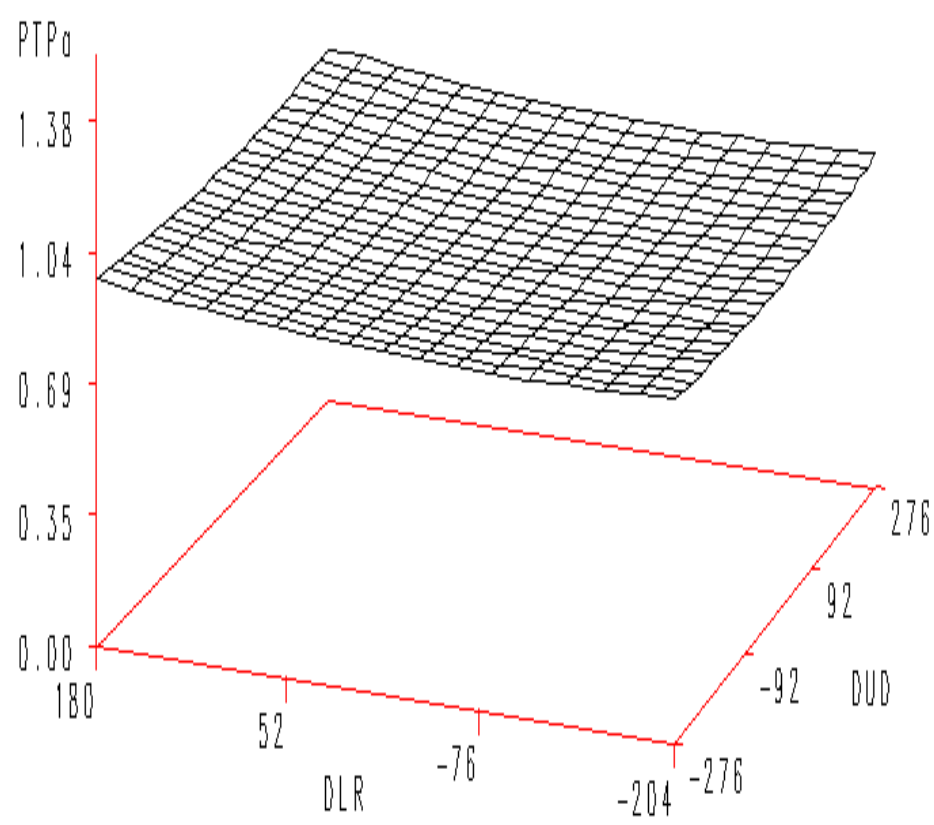

$D L R=D$ istance $(f t)$ Left to Right, DUD $=D$ istance $(f t)$ Up to Down, Up = North

Figure 6c. Fitted response surfaces of predicted total parasitoids in fields with crimson clover as the cover crop. Surfaces shown are for sampling weeks 5 and 6 . From data taken from cotton fields during the cover crop phase in Tift county, Georgia in 2001. 


\section{Cover Crop Effect on Numbers of Insects in Cotton}

Sumple Time=Cover Crop, CoverCrop=Crimson Clover PTPo = Predicted Totol Porositoids Sumpletheek=?

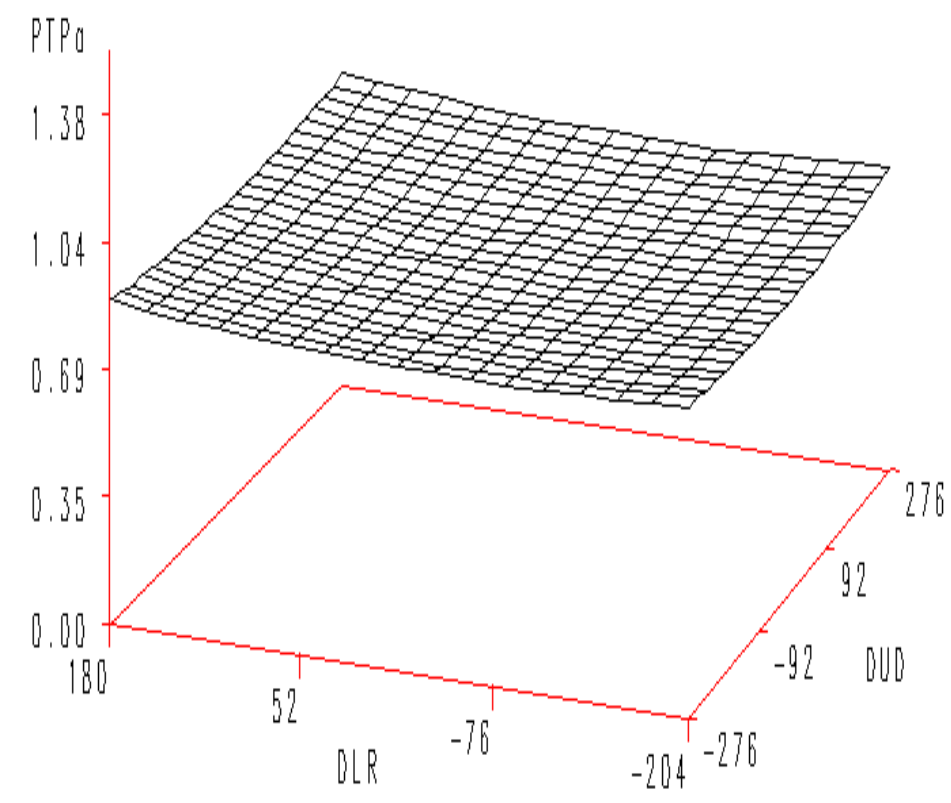

$D L R=$ Distance $(\mathrm{ft})$ Left to Riqht, DUD $=$ Distance $(\mathrm{ft})$ Up to Down, $\mathrm{Up}_{\mathrm{p}}=$ North

\section{Cover Crop Effect on Numbers of Insects in Cotton \\ Sumpletime=Cover Crop, Cover Crop=Crimson Clover \\ PTPa = Predicted Total Porositoids \\ Sompl elleek $=8$}

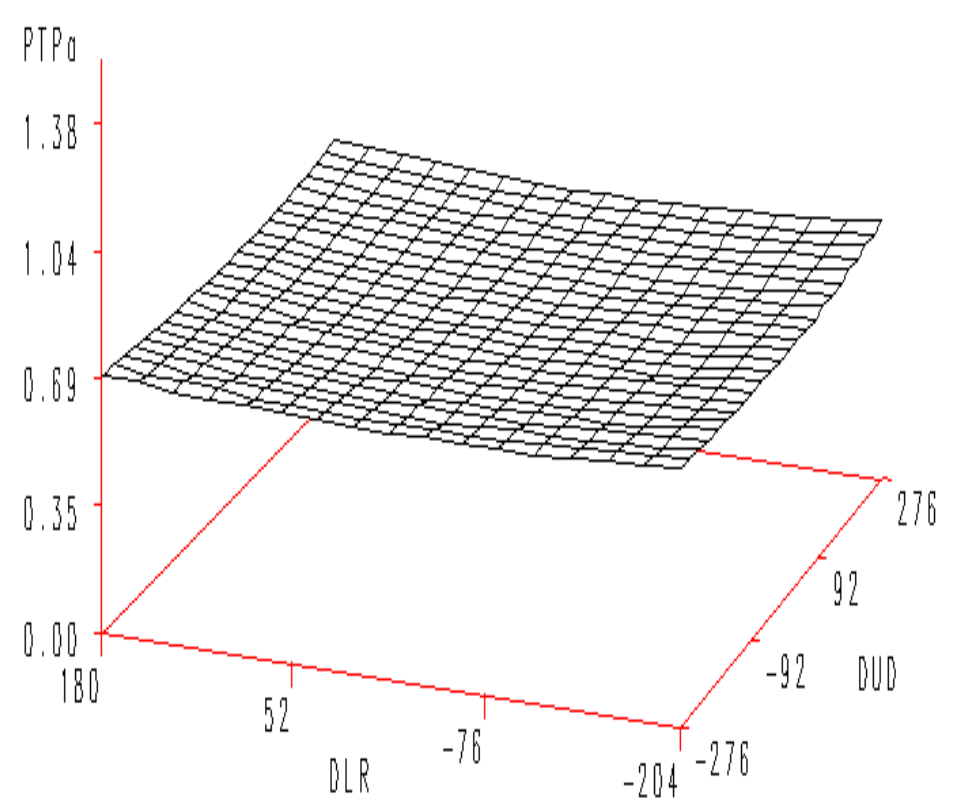

$D L R=$ Distance $(\mathrm{ft})$ Left to Right, DUD $=$ Distance $(\mathrm{ft})$ Up to Down, Up $=$ North

Figure 6d. Fitted response surfaces of predicted total parasitoids in fields with crimson clover as the cover crop. Surfaces shown are for sampling weeks 7 and 8 . From data taken from cotton fields during the cover crop phase in Tift county, Georgia in 2001. 


\section{Cover Crop Effect on Numbers of Insects in Cotton}

Sumpletime=Cover Crop, CoverCrop=Crimson Clover PTPo = Predicted Totol Porositoids Sampl elleek=9

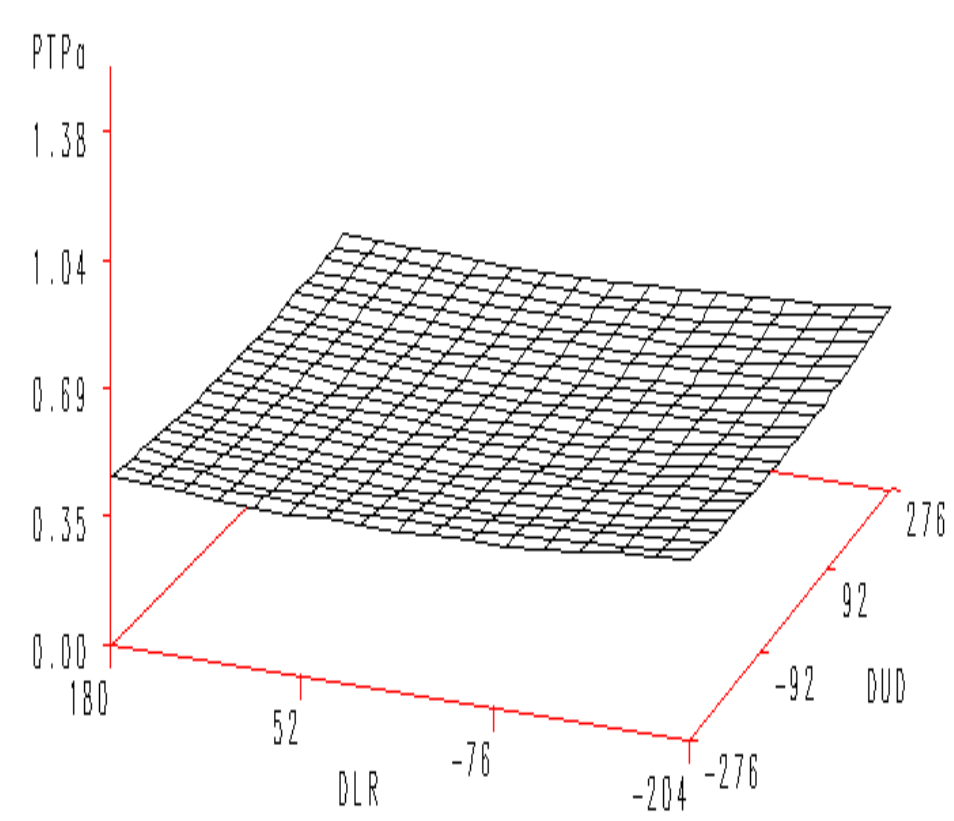

$\mathrm{DLR}=$ Distance $(\mathrm{ft})$ Left to Right, DUD $=$ Distance $(\mathrm{ft})$ Up to Down, Up $=$ North

\section{Cover Crop Effect on Numbers of Insects on Cotton}

Sampletime=Cover Crop, CoverCrop=Crimson Clover PTPo = Predicted Totol Porositoids Somp leteek $=10$

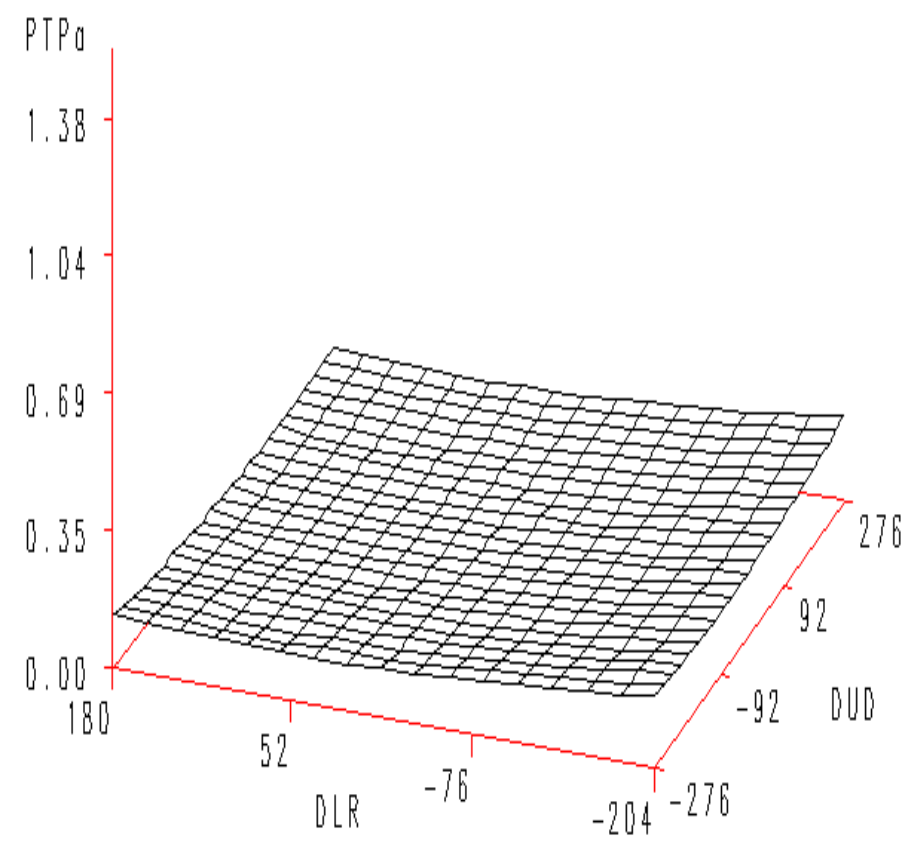

DLR $=$ Distance $(f t)$ Left to Right, DUD $=$ Distance $(f t)$ Up to Down, Up $=$ North

Figure 6e. Fitted response surfaces of predicted total parasitoids in fields with crimson clover as the cover crop. Surfaces shown are for sampling weeks 9 and 10. From data taken from cotton fields during the cover crop phase in Tift county, Georgia in 2001. 
Cover Crop Effect on Numbers of Insects in Cotton

sompletime=Cotton, CoverCrop=Crimson Clover

PTPo = Predicted Totol Porositoids

Sompletleek $=14$

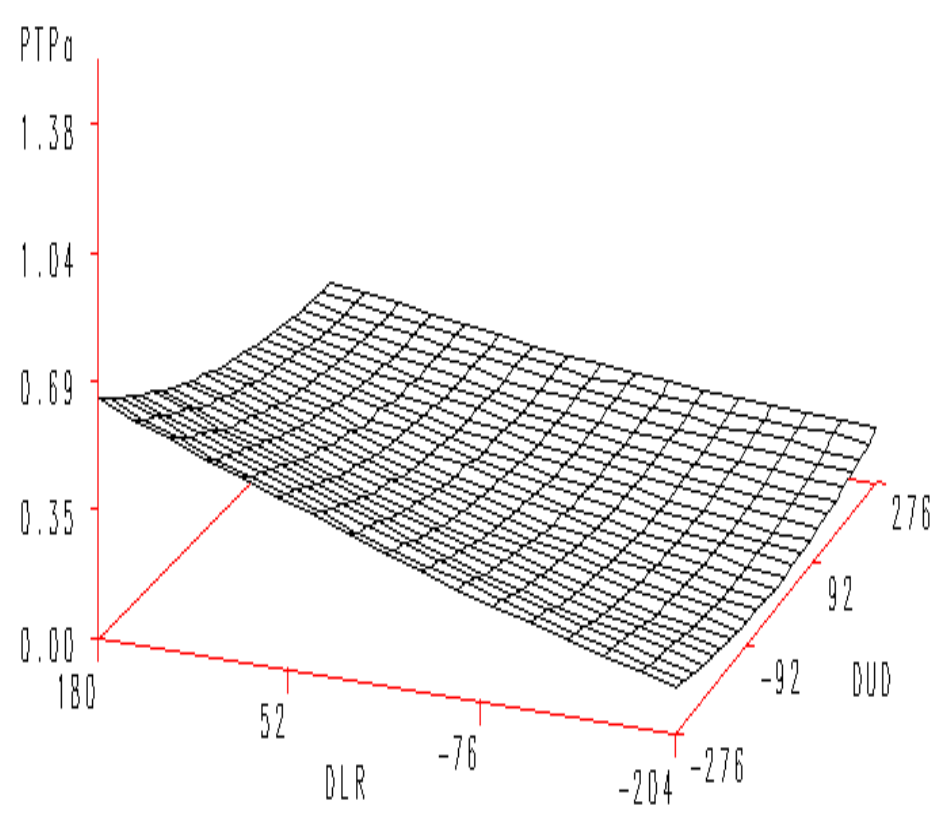

$D L R=$ Distance $(\mathrm{ft})$ Left to Riqht, DUD $=$ Distance $(\mathrm{ft})$ Up to Down, Up $=$ North

\section{Cover Crop Effect on Numbers of Insects in Cotton \\ Sampletime=Cotton, CoverCrop=Crimson Clover \\ PTPo = Predicted Total Porositoids \\ Sampletleek $=15$}

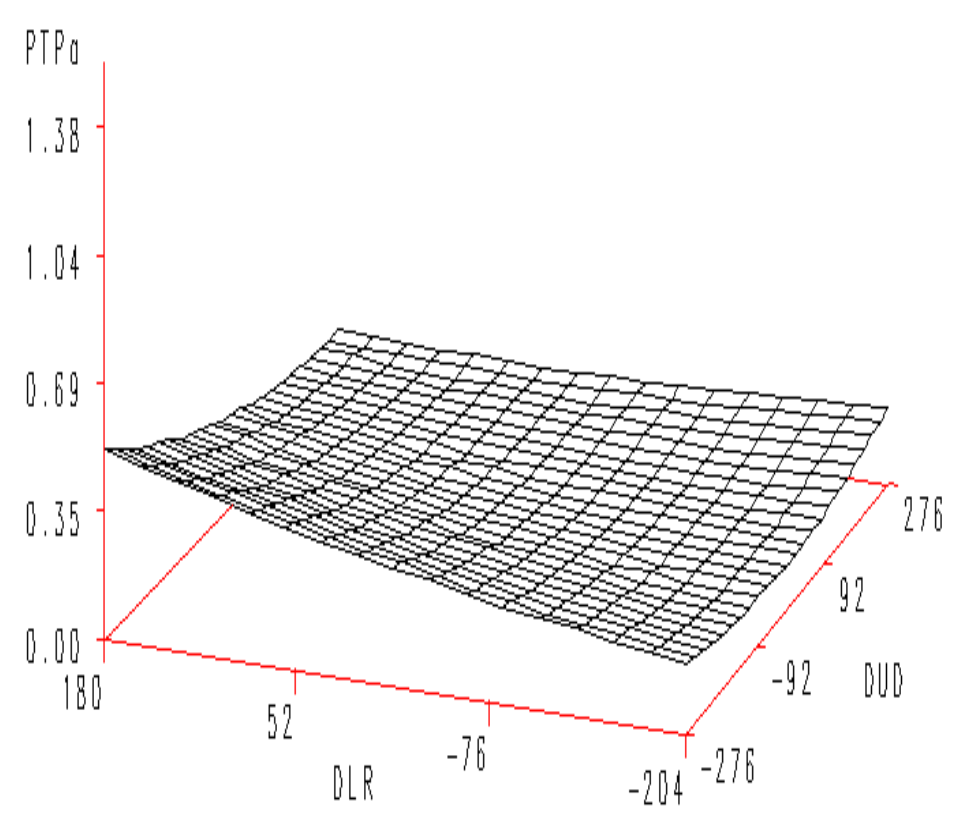

$D L R=D i s t a n c e(f t)$ Left to Riqht, DUD $=$ Distance $(f t)$ Up to Down, Up $=$ North

Figure 7a. Fitted response surfaces of predicted total parasitoids in fields with crimson clover as the cover crop. Surfaces shown are for sampling weeks 14 and 15. From data taken from cotton fields during the cotton crop phase in Tift county, Georgia in 2001. 


\section{Cover Crop Effect on Numbers of Insects on Cotton}

sumple Time =Cotton, CoverCrop=Crimson Clover

PTPO = Predicted Total Porasitoids

Somp leteek $=16$

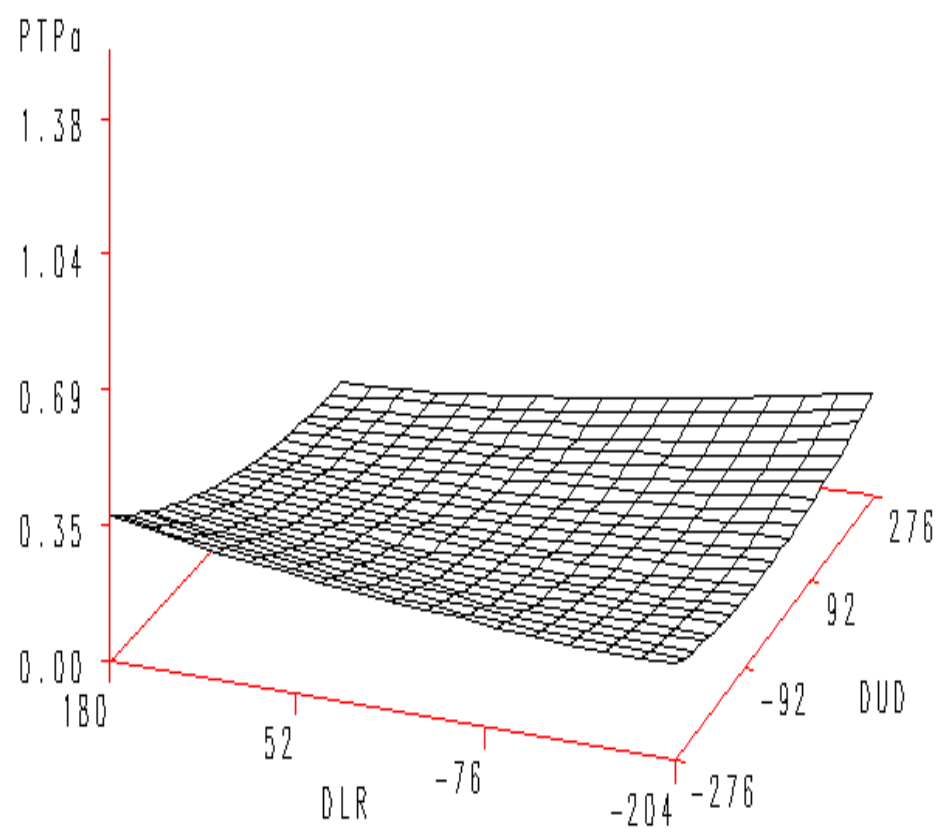

$D L R=$ Distance $(\mathrm{ft})$ Left to Riqht, DUD $=$ Distance $(\mathrm{ft})$ Up to Down, Up $=$ North

\section{Cover Crop Effect on Numbers of Insects on Cotton \\ Sumpletime=Cotton, CoverCrop=Crimson Clover \\ PTPO = Predicted Totol Porositoids Sompletheek =17}

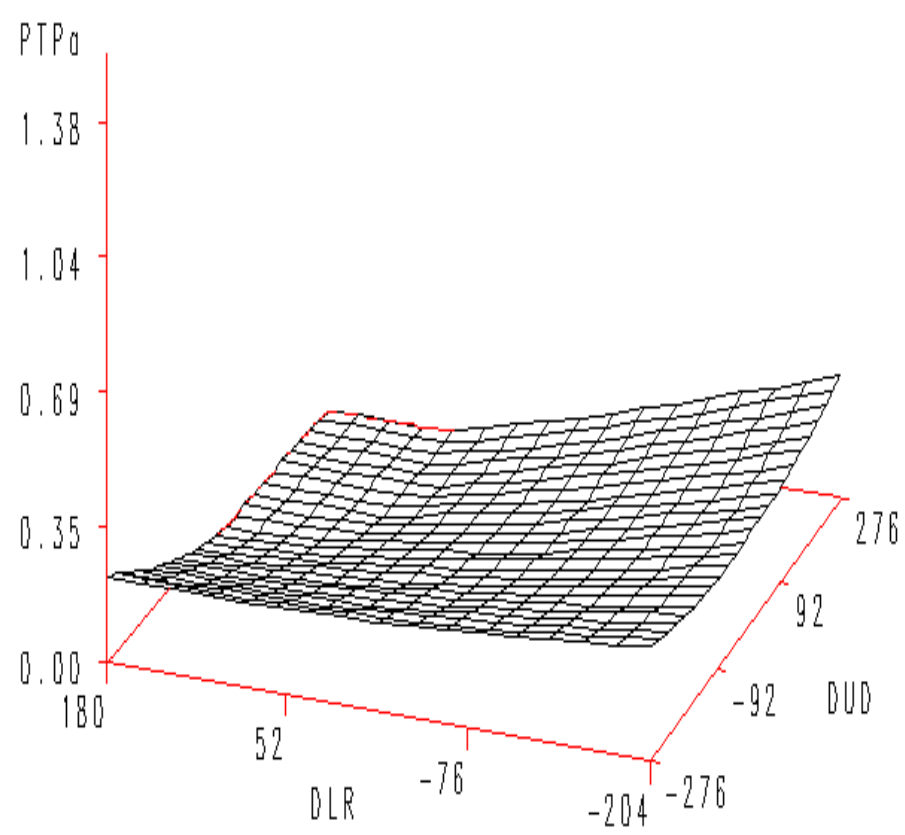

$D L R=D$ istance $(\mathrm{ft})$ Left to Riqht, DUD $=$ Distance $(\mathrm{ft})$ Up to Down, Up $=$ North

Figure 7b. Fitted response surfaces of predicted total parasitoids in fields with crimson clover as the cover crop. Surfaces shown are for sampling weeks 16 and 17. From data taken from cotton fields during the cotton crop phase in Tift county, Georgia in 2001. 


\section{Cover Crop Effect on Numbers of Insects in Cotton \\ Sanpletine:Cotton, CoverCrop=Crinson Clover \\ $P T P O=$ Predicted Totol Porositoids Sompleteek $=18$}

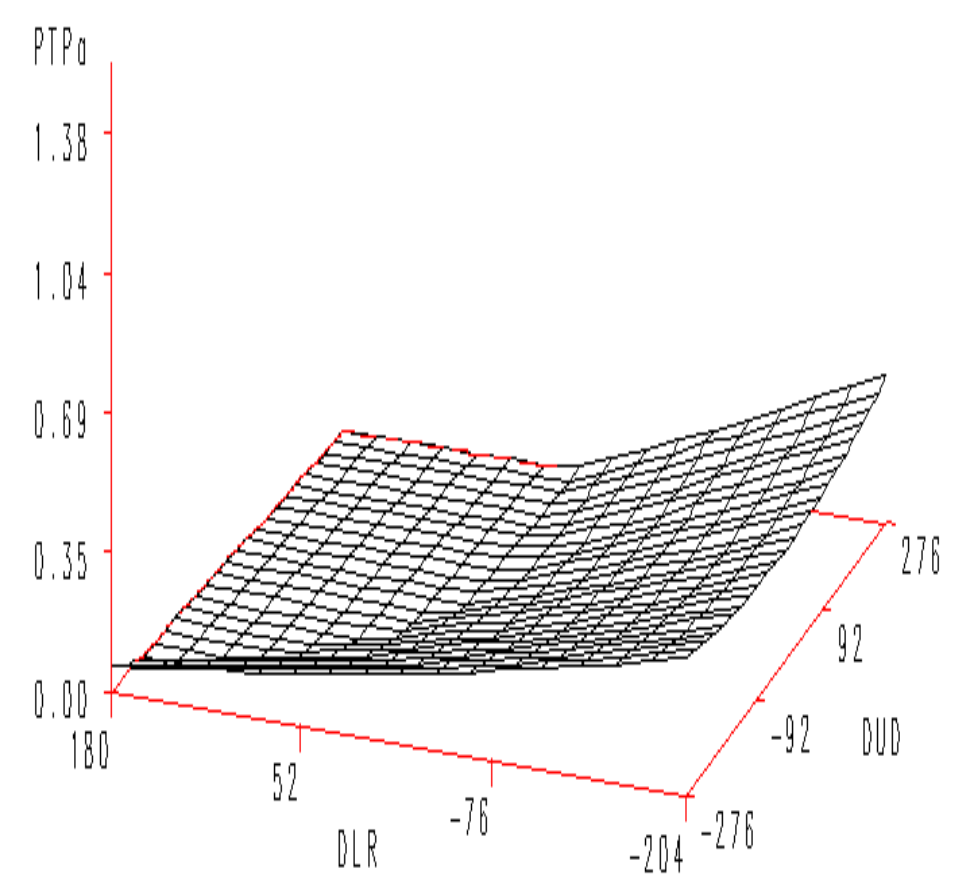

$D L R=$ Distance $(\mathrm{ft})$ Left to Riqht, DUD = Distance $(\mathrm{ft}) U_{p}$ to Down, $U_{p}=$ North
Figure 7c. Fitted response surfaces of predicted total parasitoids in fields with crimson clover as the cover crop. Surface shown is for sampling week 18. From data taken from cotton fields during the cotton crop phase in Tift county, Georgia in 2001. 
Table 3. ANOVA for Total Insect Pests during the Cover Crop Phase of the Study involving 20 farms located in Tift County, Georgia in 2001 prior to planting Cotton.

\begin{tabular}{|l|r|r|r|r|r|}
\hline Source of Variation & \multicolumn{1}{|c|}{$\mathrm{df}$} & $\mathrm{R} / \mathrm{F}$ & $\mathrm{VC} / \mathrm{MS}$ & $\mathrm{Z} / \mathrm{F}$ & $\mathrm{Pr}>\mathrm{Z} / \mathrm{F}$ \\
\hline Cover Crop (CC) & 3 & $\mathrm{R}$ & 17.1745 & 1.18 & 0.1186 \\
\hline DLR(CC) & 81 & $\mathrm{R}$ & 0.2143 & 6.34 & 0.0001 \\
\hline DUD(CC) & 93 & $\mathrm{R}$ & 0.6008 & 6.81 & 0.0001 \\
\hline SampleWeek (SW) & 7 & $\mathrm{R}$ & 0.0000 & --- & --- \\
\hline CC*SW & 27 & $\mathrm{R}$ & 5.8239 & 4.12 & 0.0001 \\
\hline mSW & 1 & $\mathrm{~F}$ & $2.065 \mathrm{e}-3$ & 0.01 & 0.9200 \\
\hline mDLR & 1 & $\mathrm{~F}$ & 11.3967 & 55.19 & 0.0001 \\
\hline mDUD & 1 & $\mathrm{~F}$ & 3.3721 & 16.33 & 0.0001 \\
\hline mSW*mSW & 1 & $\mathrm{~F}$ & 26.1470 & 126.62 & 0.0001 \\
\hline mDLR*mDLR & 1 & $\mathrm{~F}$ & 1.6892 & 8.18 & 0.0042 \\
\hline mDUD*mDUD & 1 & $\mathrm{~F}$ & 0.01032 & 0.05 & 0.8307 \\
\hline mSW*mDLR & 1 & $\mathrm{~F}$ & 0.0351 & 0.17 & 0.6804 \\
\hline mSW*mDUD & 1 & $\mathrm{~F}$ & 21.6256 & 133.78 & 0.0001 \\
\hline mDLR*mDUD & 1218 & $\mathrm{~F}$ & 39.9371 & 193.40 & 0.0001 \\
\hline mSW*mDLR*mDUD & $\mathrm{F}$ & 14.7069 & 71.22 & 0.0001 \\
\hline Residual & 1 & 0.2065 & 103.00 & 0.0001 \\
\hline
\end{tabular}

Notes: R/F denotes Random or Fixed Effects in the Proc MIXED model. VC / MS denotes Variance Component or Mean Square associated with R/F, resp. Z / F denotes the statistical test used, either the Z test (standardized Chi-Square test) or the F-test. DLR denotes the distance in feet left to right (West to East). DUD denotes the distance in feet up or down where up is North. Lower case prefix ' $m$ ' on fixed effect lines denotes that for SW, 5.5 was subtracted from each Sample Week (Draper \& Smith, 1981) and answer was divided by 2; and for DLR and DUD, distances were determined from center of field in the four cardinal directions and they were divided by 10. Magnitude of numbers were diminished to facilitate ease of computation in Proc MIXED (SAS, 2002). Insect pests include TBW (tobacco bud worm), Heliothis virescens (F.), CEW (corn ear worm), Helicoverpa zea (Boddie), TPB (tarnished plant bug), Lygus lineolaris (Palisot de Beauvois), SGSB (southern green stink bug), Nezara viridula, BSB (brown stink bug), Euschistus servus (Say), and GSB (green stink bug), Acrosternum hilare (Say). 
Table 4. ANOVA for Total Insect Pests during the Cotton Phase of the Study involving 20 farms located in Tift County, Georgia in 2001 after Cotton was Planted..

\begin{tabular}{|c|c|c|c|c|c|}
\hline Source of Variation & df & $\mathrm{R} / \mathrm{F}$ & VC / MS & $\mathrm{Z} / \mathrm{F}$ & $\operatorname{Pr}>\mathrm{Z} / \mathrm{F}$ \\
\hline Cover Crop (CC) & 4 & $\mathrm{R}$ & 9.9699 & 0.87 & 0.1914 \\
\hline DLR(CC) & 108 & $\mathrm{R}$ & 0.3707 & 7.10 & 0.0001 \\
\hline DUD(CC) & 120 & $\mathrm{R}$ & 1.0247 & 7.62 & 0.0001 \\
\hline SampleWeek (SW) & 2 & $\mathrm{R}$ & 0.0000 & --- & --- \\
\hline $\mathrm{CC} * \mathrm{SW}$ & 16 & $\mathrm{R}$ & 29.4368 & 3.00 & 0.0014 \\
\hline mSW & 1 & $\mathrm{~F}$ & 19.0671 & 11.56 & 0.0007 \\
\hline mDLR & 1 & $\mathrm{~F}$ & 0.4124 & 0.25 & 0.6188 \\
\hline mDUD & 1 & $\mathrm{~F}$ & 86.1647 & 52.24 & 0.0001 \\
\hline $\mathrm{mSW}^{*} \mathrm{mSW}$ & 1 & $\mathrm{~F}$ & 6.1358 & 3.72 & 0.0539 \\
\hline $\mathrm{mDLR} * \mathrm{mDLR}$ & 1 & $\mathrm{~F}$ & 105.3967 & 63.90 & 0.0001 \\
\hline mDUD*mDUD & 1 & $\mathrm{~F}$ & 40.5917 & 24.61 & 0.0001 \\
\hline $\mathrm{mSW}{ }^{*} \mathrm{mDLR}$ & 1 & $\mathrm{~F}$ & 48.7398 & 29.55 & 0.0001 \\
\hline mSW*mDUD & 1 & $\mathrm{~F}$ & 3464.9935 & 2100.76 & 0.0001 \\
\hline mDLR*mDUD & 1 & $\mathrm{~F}$ & 895.5747 & 542.97 & 0.0001 \\
\hline $\mathrm{mSW}{ }^{*} \mathrm{mDLR}{ }^{*} \mathrm{mDUD}$ & 1 & $\mathrm{~F}$ & 0.1320 & 0.08 & 0.7720 \\
\hline Residual & 14379 & $\mathrm{R}$ & 1.6494 & 84.79 & 0.0001 \\
\hline
\end{tabular}

Notes: R/F denotes Random or Fixed Effects in the Proc MIXED model. VC / MS denotes Variance Component or Mean Square associated with R/F, resp. Z / F denotes the statistical test used, either the Z test (standardized Chi-Square test) or the F-test. DLR denotes the distance in feet left to right (West to East). DUD denotes the distance in feet up or down where up is North. Lower case prefix ' $m$ ' on fixed effect lines denotes that for SW, 16 was subtracted from each Sample Week (Draper \& Smith, 1981) and answer was divided by 2; and for DLR and DUD, distances were determined from center of field in the four cardinal directions and they were divided by 10. Magnitude of numbers were diminished to facilitate ease of computation in Proc MIXED (SAS, 2002). Insect pests include TBW (tobacco bud worm), Heliothis virescens (F.), CEW (corn ear worm), Helicoverpa zea (Boddie), TPB (tarnished plant bug), Lygus lineolaris (Palisot de Beauvois), SGSB (southern green stink bug), Nezara viridula, BSB (brown stink bug), Euschistus servus (Say), and GSB (green stink bug), Acrosternum hilare (Say). 
Table 5. ANOVA for Total Insect Predators during the Cover Crop Phase of the Study involving 20 farms located in Tift County, Georgia in 2001 prior to planting Cotton.

\begin{tabular}{|c|c|c|c|c|c|}
\hline Source of Variation & $\mathrm{df}$ & $\mathrm{R} / \mathrm{F}$ & VC / MS & $\mathrm{Z} / \mathrm{F}$ & $\operatorname{Pr}>\mathrm{Z} / \mathrm{F}$ \\
\hline Cover Crop (CC) & 3 & $\mathrm{R}$ & 10.3221 & 1.19 & 0.1163 \\
\hline $\operatorname{DLR}(\mathrm{CC})$ & 81 & $\mathrm{R}$ & 0.0134 & 6.33 & 0.0001 \\
\hline DUD(CC) & 93 & $\mathrm{R}$ & 0.0287 & 6.80 & 0.0001 \\
\hline SampleWeek (SW) & 7 & $\mathrm{R}$ & 0.0000 & --- & --- \\
\hline $\mathrm{CC} * \mathrm{SW}$ & 27 & $\mathrm{R}$ & 2.6495 & 4.12 & 0.0001 \\
\hline $\mathrm{mSW}$ & 1 & $\mathrm{~F}$ & 0.1128 & 5.79 & 0.0161 \\
\hline mDLR & 1 & $\mathrm{~F}$ & 1.0562 & 54.22 & 0.0001 \\
\hline mDUD & 1 & $\mathrm{~F}$ & 0.3767 & 19.34 & 0.0001 \\
\hline $\mathrm{mSW}^{*} \mathrm{mSW}$ & 1 & $\mathrm{~F}$ & 0.7724 & 39.65 & 0.0001 \\
\hline $\mathrm{mDLR} * \mathrm{mDLR}$ & 1 & $\mathrm{~F}$ & 0.0592 & 3.04 & 0.0813 \\
\hline mDUD*mDUD & 1 & $\mathrm{~F}$ & $6.039 \mathrm{e}-3$ & 0.31 & 0.5769 \\
\hline $\mathrm{mSW} * \mathrm{mDLR}$ & 1 & $\mathrm{~F}$ & 3.3712 & 173.06 & 0.0001 \\
\hline $\mathrm{mSW} * \mathrm{mDUD}$ & 1 & $\mathrm{~F}$ & 6.0626 & 311.22 & 0.0001 \\
\hline mDLR*mDUD & 1 & $\mathrm{~F}$ & 14.2282 & 730.40 & 0.0001 \\
\hline $\mathrm{mSW} * \mathrm{mDLR} * \mathrm{mDUD}$ & 1 & $\mathrm{~F}$ & 0.1126 & 5.78 & 0.0162 \\
\hline Residual & 21218 & $\mathrm{R}$ & 0.0195 & 103.00 & 0.0001 \\
\hline
\end{tabular}

Notes: R/F denotes Random or Fixed Effects in the Proc MIXED model. VC / MS denotes Variance Component or Mean Square associated with R/F, resp. Z / F denotes the statistical test used, either the $\mathrm{Z}$ test (standardized Chi-Square test) or the F-test. DLR denotes the distance in feet left to right (West to East). DUD denotes the distance in feet up or down where up is North. Lower case prefix ' $m$ ' on fixed effect lines denotes that for SW, 5.5 was subtracted from each Sample Week (Draper \& Smith, 1981) and answer was divided by 2; and for DLR and DUD, distances were determined from center of field in the four cardinal directions and they were divided by 10. Magnitude of numbers were diminished to facilitate ease of computation in Proc MIXED (SAS, 2002). Insect Predators include big-eyed bug, Geocoris punctipes (Say); pirate bug, Orius insidiosus (Say); red imported fire ant, Solenopsis invicta Buren; and these four lady bugs: convergent lady beetle, Hippodamia convergens Guerin-Meneville; sevenspotted lady beetle, Coccinella septempunctata L.; ladybird beetle, Coleomegilla maculata (DeGeer); and multicolored Asian lady beetle, Harmonia axyridis (Pallas). 
Table 6. ANOVA for Total Insect Predators during the Cotton Phase of the Study involving 20 farms located in Tift County, Georgia in 2001 after Cotton was Planted..

\begin{tabular}{|c|c|c|c|c|c|}
\hline Source of Variation & $\mathrm{df}$ & $\mathrm{R} / \mathrm{F}$ & VC / MS & $\mathrm{Z} / \mathrm{F}$ & $\operatorname{Pr}>\mathrm{Z} / \mathrm{F}$ \\
\hline Cover Crop (CC) & 4 & $\mathrm{R}$ & 2.1038 & 1.27 & 0.1012 \\
\hline $\operatorname{DLR}(\mathrm{CC})$ & 108 & $\mathrm{R}$ & 0.0769 & 7.32 & 0.0001 \\
\hline DUD(CC) & 120 & $\mathrm{R}$ & 0.0417 & 7.69 & 0.0001 \\
\hline SampleWeek (SW) & 2 & $\mathrm{R}$ & 0.0000 & --- & --- \\
\hline $\mathrm{CC} * \mathrm{SW}$ & 16 & $\mathrm{R}$ & 1.1146 & 3.00 & 0.0014 \\
\hline $\mathrm{mSW}$ & 1 & $\mathrm{~F}$ & 0.3712 & 11.12 & 0.0009 \\
\hline mDLR & 1 & $\mathrm{~F}$ & 0.0407 & 1.22 & 0.2695 \\
\hline mDUD & 1 & $\mathrm{~F}$ & 0.8108 & 24.29 & 0.0001 \\
\hline $\mathrm{mSW}^{*} \mathrm{mSW}$ & 1 & $\mathrm{~F}$ & 0.0110 & 0.30 & 0.5826 \\
\hline $\mathrm{mDLR} * \mathrm{mDLR}$ & 1 & $\mathrm{~F}$ & 0.1198 & 3.59 & 0.0583 \\
\hline mDUD*mDUD & 1 & $\mathrm{~F}$ & 0.0664 & 1.99 & 0.1579 \\
\hline $\mathrm{mSW} * \mathrm{mDLR}$ & 1 & $\mathrm{~F}$ & 121.8650 & 3650.84 & 0.0001 \\
\hline $\mathrm{mSW}^{*} \mathrm{mDUD}$ & 1 & $\mathrm{~F}$ & 20.5948 & 616.98 & 0.0001 \\
\hline mDLR*mDUD & 1 & $\mathrm{~F}$ & 44.4094 & 1330.42 & 0.0001 \\
\hline $\mathrm{mSW}^{*} \mathrm{mDLR}{ }^{*} \mathrm{mDUD}$ & 1 & $\mathrm{~F}$ & 0.0174 & 0.52 & 0.4723 \\
\hline Residual & 14379 & $\mathrm{R}$ & 0.0334 & 84.79 & 0.0001 \\
\hline
\end{tabular}

Notes: R/F denotes Random or Fixed Effects in the Proc MIXED model. VC / MS denotes Variance Component or Mean Square associated with R/F, resp. Z / F denotes the statistical test used, either the $\mathrm{Z}$ test (standardized Chi-Square test) or the F-test. DLR denotes the distance in feet left to right (West to East). DUD denotes the distance in feet up or down where up is North. Lower case prefix ' $m$ ' on fixed effect lines denotes that for SW, 16 was subtracted from each Sample Week (Draper \& Smith, 1981) and answer was divided by 2; and for DLR and DUD, distances were determined from center of field in the four cardinal directions and they were divided by 10. Magnitude of numbers were diminished to facilitate ease of computation in Proc MIXED (SAS, 2002). Insect Predators include big-eyed bug, Geocoris punctipes (Say); pirate bug, Orius insidiosus (Say); red imported fire ant, Solenopsis invicta Buren; and these four lady bugs: convergent lady beetle, Hippodamia convergens Guerin-Meneville; sevenspotted lady beetle, Coccinella septempunctata L.; ladybird beetle, Coleomegilla maculata (DeGeer); and multicolored Asian lady beetle, Harmonia axyridis (Pallas). 
Table 7. ANOVA for Total Insect Parasitoids during the Cover Crop Phase of the Study involving 20 farms located in Tift County, Georgia in 2001 prior to planting Cotton.

\begin{tabular}{|c|c|c|c|c|c|}
\hline Source of Variation & $\mathrm{df}$ & $\mathrm{R} / \mathrm{F}$ & $\mathrm{VC} / \mathrm{MS}$ & $\mathrm{Z} / \mathrm{F}$ & $\operatorname{Pr}>\mathrm{Z} / \mathrm{F}$ \\
\hline Cover Crop (CC) & 3 & $\mathrm{R}$ & 0.0691 & 1.19 & 0.1172 \\
\hline $\operatorname{DLR}(\mathrm{CC})$ & 81 & $\mathrm{R}$ & $1.958 \mathrm{e}-3$ & 6.33 & 0.0001 \\
\hline DUD(CC) & 93 & $\mathrm{R}$ & $7.82 \mathrm{e}-4$ & 6.70 & 0.0001 \\
\hline SampleWeek (SW) & 7 & $\mathrm{R}$ & 0.0000 & --- & --- \\
\hline $\mathrm{CC} * \mathrm{SW}$ & 27 & $\mathrm{R}$ & 0.0194 & 4.12 & 0.0001 \\
\hline $\mathrm{mSW}$ & 1 & $\mathrm{~F}$ & $4.634 \mathrm{e}-3$ & 1.61 & 0.2042 \\
\hline mDLR & 1 & $\mathrm{~F}$ & 0.1553 & 53.96 & 0.0001 \\
\hline mDUD & 1 & $\mathrm{~F}$ & $8.92 \mathrm{e}-4$ & 0.31 & 0.5801 \\
\hline $\mathrm{mSW}^{*} \mathrm{mSW}$ & 1 & $\mathrm{~F}$ & 0.3408 & 118.4 & 0.0001 \\
\hline mDLR*mDLR & 1 & $\mathrm{~F}$ & 0.0355 & 12.32 & 0.0004 \\
\hline mDUD*mDUD & 1 & $\mathrm{~F}$ & 0.0521 & 18.09 & 0.0001 \\
\hline $\mathrm{mSW} * \mathrm{mDLR}$ & 1 & $\mathrm{~F}$ & 4.2441 & 1474.66 & 0.0001 \\
\hline $\mathrm{mSW}{ }^{*} \mathrm{mDUD}$ & 1 & $\mathrm{~F}$ & 0.1837 & 63.82 & 0.0001 \\
\hline mDLR*mDUD & 1 & $\mathrm{~F}$ & 4.6388 & 1611.81 & 0.0001 \\
\hline $\mathrm{mSW}^{*} \mathrm{mDLR} * \mathrm{mDUD}$ & 1 & $\mathrm{~F}$ & 0.0482 & 16.73 & 0.0001 \\
\hline Residual & 21218 & $\mathrm{R}$ & $2.878 \mathrm{e}-3$ & 103.00 & 0.0001 \\
\hline
\end{tabular}

Notes: R/F denotes Random or Fixed Effects in the Proc MIXED model. VC / MS denotes Variance Component or Mean Square associated with R/F, resp. Z / F denotes the statistical test used, either the $\mathrm{Z}$ test (standardized Chi-Square test) or the F-test. DLR denotes the distance in feet left to right (West to East). DUD denotes the distance in feet up or down where up is North. Lower case prefix ' $m$ ' on fixed effect lines denotes that for SW, 5.5 was subtracted from each Sample Week (Draper \& Smith, 1981) and answer was divided by 2; and for DLR and DUD, distances were determined from center of field in the four cardinal directions and they were divided by 10. Magnitude of numbers were diminished to facilitate ease of computation in Proc MIXED (SAS, 2002). Evidence of parasitation was determined by examining those insect pests found to see if they had a puncture mark on their bodies. 
Table 8. ANOVA for Total Insect Parasitoids during the Cotton Phase of the Study involving 20 farms located in Tift County, Georgia in 2001 after Cotton was Planted..

\begin{tabular}{|c|c|c|c|c|c|}
\hline Source of Variation & $\mathrm{df}$ & $\mathrm{R} / \mathrm{F}$ & $\mathrm{VC} / \mathrm{MS}$ & $\mathrm{Z} / \mathrm{F}$ & $\operatorname{Pr}>\mathrm{Z} / \mathrm{F}$ \\
\hline Cover Crop (CC) & 4 & $\mathrm{R}$ & 0.9289 & 1.40 & 0.0802 \\
\hline $\operatorname{DLR}(\mathrm{CC})$ & 108 & $\mathrm{R}$ & 0.0442 & 7.34 & 0.0001 \\
\hline DUD(CC) & 120 & $\mathrm{R}$ & 0.0223 & 7.73 & 0.0001 \\
\hline SampleWeek (SW) & 2 & $\mathrm{R}$ & 0.0000 & --- & --- \\
\hline $\mathrm{CC} * \mathrm{SW}$ & 16 & $\mathrm{R}$ & 0.0218 & 3.00 & 0.0014 \\
\hline $\mathrm{mSW}$ & 1 & $\mathrm{~F}$ & $8.27 e-4$ & 0.18 & 0.6692 \\
\hline mDLR & 1 & $\mathrm{~F}$ & 0.0345 & 7.50 & 0.0062 \\
\hline mDUD & 1 & $\mathrm{~F}$ & $9.689 e-3$ & 2.11 & 0.1465 \\
\hline $\mathrm{mSW}^{*} \mathrm{mSW}$ & 1 & $\mathrm{~F}$ & $9.414 \mathrm{e}-3$ & 2.05 & 0.1522 \\
\hline $\mathrm{mDLR} * \mathrm{mDLR}$ & 1 & $\mathrm{~F}$ & 0.2341 & 50.98 & 0.0001 \\
\hline mDUD*mDUD & 1 & $\mathrm{~F}$ & 0.3235 & 70.44 & 0.0001 \\
\hline $\mathrm{mSW}^{*} \mathrm{mDLR}$ & 1 & $\mathrm{~F}$ & 16.7708 & 3652.18 & 0.0001 \\
\hline $\mathrm{mSW}{ }^{*} \mathrm{mDUD}$ & 1 & $\mathrm{~F}$ & 0.9253 & 201.49 & 0.0001 \\
\hline mDLR*mDUD & 1 & $\mathrm{~F}$ & 28.7616 & 6263.42 & 0.0001 \\
\hline $\mathrm{mSW}^{*} \mathrm{mDLR} * \mathrm{mDUD}$ & 1 & $\mathrm{~F}$ & 0.0468 & 10.19 & 0.0014 \\
\hline Residual & 14379 & $\mathrm{R}$ & $4.592 \mathrm{e}-3$ & 84.79 & 0.0001 \\
\hline
\end{tabular}

Notes: R/F denotes Random or Fixed Effects in the Proc MIXED model. VC / MS denotes Variance Component or Mean Square associated with R/F, resp. Z / F denotes the statistical test used, either the $\mathrm{Z}$ test (standardized Chi-Square test) or the F-test. DLR denotes the distance in feet left to right (West to East). DUD denotes the distance in feet up or down where up is North. Lower case prefix 'm' on fixed effect lines denotes that for SW, 16 was subtracted from each Sample Week (Draper \& Smith, 1981) and answer was divided by 2; and for DLR and DUD, distances were determined from center of field in the four cardinal directions and they were divided by 10. Magnitude of numbers were diminished to facilitate ease of computation in Proc MIXED (SAS, 2002). Evidence of parasitation was determined by examining those insect pests found to see if they had a puncture mark on their bodies. 